\title{
Advances on Graphene-Based Nanomaterials and Mesenchymal Stem Cell-Derived Exosomes Applied in Cutaneous Wound Healing
}

This article was published in the following Dove Press journal: International Journal of Nanomedicine

\author{
Ming Zhao \\ Jihong Shi \\ Weixia Cai \\ Kaituo Liu \\ Kuo Shen \\ Zichao Li \\ Yunchuan Wang (D) \\ Dahai Hu $\mathbb{D}$
}

Department of Burns and Cutaneous Surgery, Xijing Hospital, Fourth Military Medical University, Xi'an, Shannxi,

710032, People's Republic of China
Correspondence: Yunchuan Wang; Dahai $\mathrm{Hu}$

Department of Burns and Cutaneous Surgery, Xijing Hospital, Fourth Military Medical University, Xi'an, Shannxi, 710032, People's Republic of China Tel +86 29-84775293; +86 29-84775298 Email wangyunchuan@fmmu.edu.cn; hudahaidoc@163.com

\begin{abstract}
Graphene is a new type of carbon nanomaterial discovered after fullerene and carbon nanotube. Due to the excellent biological properties such as biocompatibility, cell proliferation stimulating, and antibacterial properties, graphene and its derivatives have become emerging candidates for the development of novel cutaneous wound dressings and composite scaffolds. On the other hand, pre-clinical research on exosomes derived from mesenchymal stem cells (MSC-Exos) has been intensified for cell-free treatment in wound healing and cutaneous regeneration, via ameliorating the damaged microenvironment of the wound site. Here, we provide a comprehensive understanding of the latest studies and observations on the various effects of graphene-based nanomaterials (GBNs) and MSCExos during the cutaneous wound repair process, as well as the putative mechanisms thereof. In addition, we propose the possible forward directions of GBNs and MSC-Exos applications, expecting to promote the clinical transformation.
\end{abstract}

Keywords: graphene, carbon nanomaterials, mesenchymal stem cells, exosomes, wound healing

\section{Introduction}

Graphene-based nanomaterials (GBNs), as a group of next-generation nanomaterials, have offered tremendous potential in a diverse range of applications. ${ }^{1}$ Since firstly prepared by mechanical exfoliation in $2004,{ }^{2}$ graphene has been widely used in flexible supercapacitors, electrochemical sensors and batteries, etc. ${ }^{3-6}$ In recent years, due to its excellent electronic, thermal and optical properties, graphene has attracted enormous interest. ${ }^{7-9}$ The graphene plasmons could couple with other plasmons and phonon polaritons, leading to a further mediation of near-field radiative heat transfer. ${ }^{10}$ Moreover, graphene is expected to become an ideal membrane material to separate gases, liquids and ions in terms of the selectivity and permeability, which could outperform the established polymer membranes. ${ }^{11,12}$

Likewise, a growing number of studies have also focused on the application of graphene and its derivatives in the field of biomedicine, which is in full swing and involves drug carriers, biological detection, tumor treatment and tissue engineering. ${ }^{13}$ Investigations on the $2 \mathrm{D}$ or $3 \mathrm{D}$ scaffold for cell culture suggest the ability of GBNs for mimicking in vivo environment.

Under normal circumstances, cutaneous wound healing is a rapid and efficient process leading to the reconstruction of barrier function. ${ }^{14}$ But the repair process 
may be delayed by systemic or local factors, incurring significant costs and loss of life quality to the patients involved. ${ }^{15,16}$ Various types of novel bioactive skin substitutes and cell-based therapies have emerged continually in pre-clinical and clinical studies, providing promising ideas for the repair of large-area or difficult-to-heal wounds. ${ }^{17}$ All prokaryotes and eukaryotes are able to release extracellular vesicles (EVs) under physiological or pathological conditions. Exosomes are a subset of EVs that originate from the endosome, the diameter of which ranges from 40 to $160 \mathrm{~nm}$ (average $100 \mathrm{~nm}$ ). ${ }^{18}$ The diverse constituents generally include protein, miRNA, mRNA, DNA and lipid, and participate in intercellular communication. ${ }^{19,20}$ Studies have found that the application of exosomes, especially which come from mesenchymal stem cells (MSCs), can effectively repair the cutaneous wound and achieve the promising healing. ${ }^{21}$

In this review, we focus on the booming application of graphene-based materials and MSC-Exos in cutaneous wound repair. Besides, the structure and synthesis methods of GBNs are briefly summarized, and then we highlight their excellent biological properties and biocompatibility. Finally, we present our perspectives on the potent synergistic effect between GBNs and MSC-Exos for the treatment of cutaneous wound. We also put forward the potential challenges and opportunities facing in future research.

\section{Cutaneous Wound Healing}

The cutaneous wound healing process is divided into four overlapping phases, defined as hemostasis, inflammation, proliferation and tissue matrix remodeling. ${ }^{22}$ Dysregulation of any stage would increase the risk of chronic refractory cutaneous wounds, with growing morbidity and mortality. ${ }^{23}$ After the skin is injured, local tissue initiates necrosis and bleeding. Next, blood vessels constrict and blood clots form, providing a basic environment for the subsequent wound healing. ${ }^{24}$ The blood clots can attract platelets, growth factors, cytokines, neutrophils, macrophages and endothelial cells, which collaborate well to form blood scabs so as to protect the wound, until the blood vessels dilate. ${ }^{25}$ Coagulation factors play a vital role in the infiltration of relevant cells, typically the macrophages. The mice of the hemophilia B model exhibit an impaired healing of skins, articular cartilages and bones. $^{26}$ The hemostatic protein von Willebrand factor (VWF) released by endothelial cells has been shown to regulate angiogenesis, and VWF deficiency in mice causes delayed wound healing as well as decreased angiogenesis and decreased amounts of angiogenic growth factors in the wound. ${ }^{27}$

Cutaneous injury leads to the prompt activation of a topical inflammatory response. Moderate inflammation is a highly regulated process orchestrated by resident cells and immune cells recruited spatiotemporally, fighting against infection and removing dead cells and cell debris $^{28,29}$ (Figure 1). On contrary, immoderate inflammation causes prolonged wound healing. Several kinds of innate immune cells play a pivotal role in this stage. ${ }^{30}$ The proper termination of inflammatory cascade is important to allow the execution of tissue repair and restoration of normal tissue function. Neutrophils are the first responders to epithelial injury. They infiltrate into wound tissue, clear bacteria, limit infection, and secrete proinflammatory TNF- $\alpha$, which stimulates fibroblast proliferation and angiogenesis. $^{28,31}$ Landén et al reviewed that Omega-6 Fatty Acids presented the capacity to improve wound healing due to antioxidant and anti-inflammatory effects on the inflammatory phase of tissue repair. ${ }^{32}$

Three to ten days after the wound, the healing program enters the phase of proliferation. In this phase, reepithelialization and angiogenesis overlap leading to the formation of healthy and vascularized granulation tissue. Owing to ischemia and hypoxia, the proliferation and migration capacity of dermal fibroblasts decreased and incurred refractory wounds. Transition from inflammation to proliferation is a critical step during wound healing. Factors that impact this phase transition involved macrophages, redox signals and TLR signaling. ${ }^{32} \mathrm{Li}$ et a ${ }^{33}$ proved that inhibition of miR-132 in keratinocytes may delay the transition from the inflammatory to the proliferative phase during wound healing. Single-cell RNA-sequencing analysis distinguished differential subpopulations of dermal fibroblasts ${ }^{34}$ with heterogenous function. ${ }^{35}$ The shift in fibroblast composition influenced wound healing rate in vivo. TNF signaling and several other intrinsic and extrinsic cytokines seemed key contributing factors to the variability in ageing phenotypes, including wound healing, and even detrimental fibrosis in vivo. ${ }^{34}$

Extracellular matrix remodeling and scar formation are the final stages of wound closure. It begins 2 to 3 weeks following the initial injury and lasts up to a year or more, depending on wound severity. The imbalance in synthesis and degradation of extracellular matrix may lead to wound non-healing or pathological scar formation. ${ }^{36,37}$ The pro-inflammatory cytokine TNF- $\alpha$ enhances collagenolysis and increases the activation of MMP-13 by up-regulating MMP- $3 .^{38}$ Compared to the 


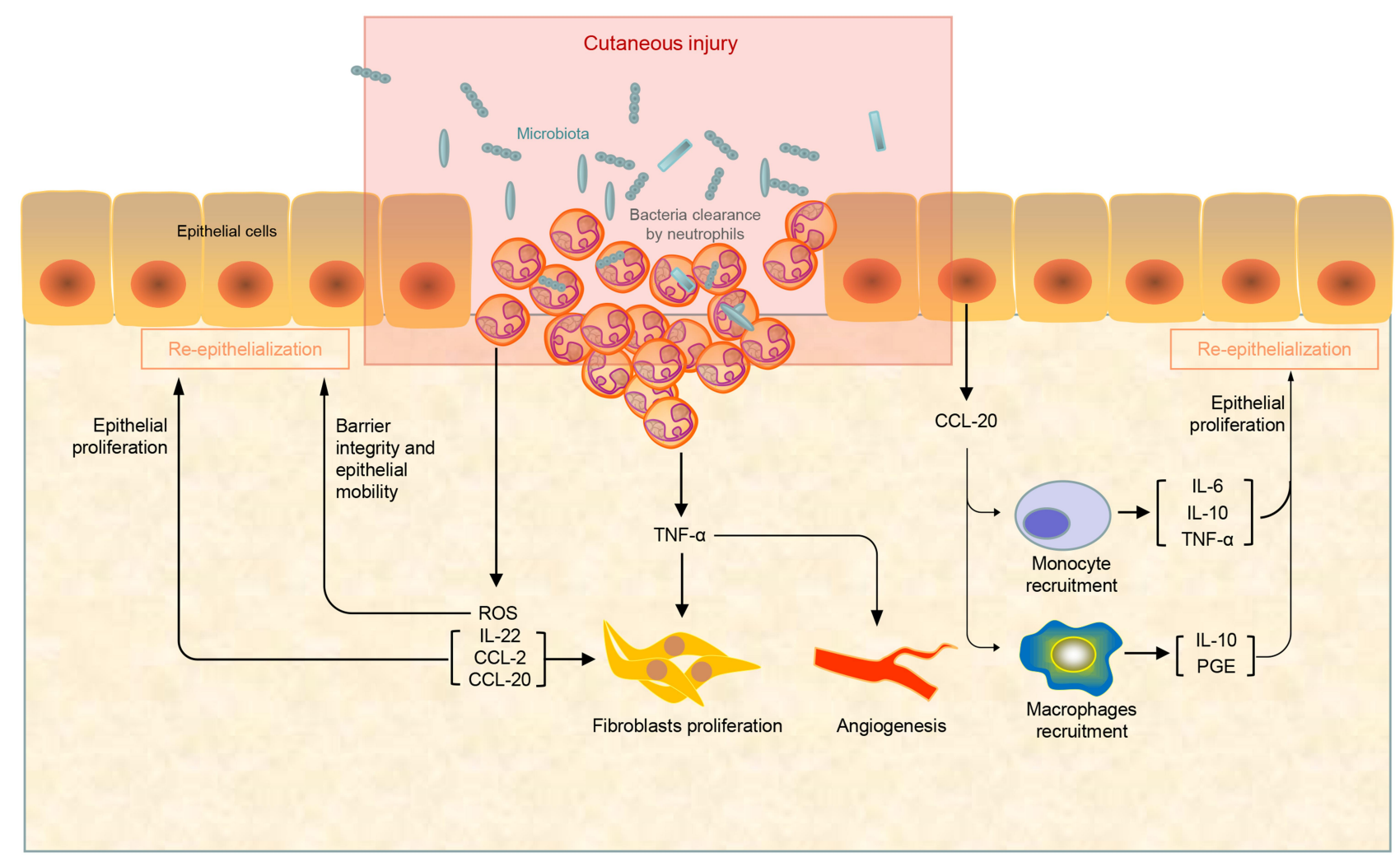

Figure I The proinflammatory stage of wound healing. Neutrophils respond quickly once the cutaneous injury happened to limit bacterial infections at the wound site and stimulates fibroblast proliferation and angiogenesis by the secretion of series of cytokines.

physiologic scarring in adults, fetal cutaneous wound healing is uniquely characterized by repair with complete restoration of dermal architecture. Microarray analysis revealed that signaling pathways, like proline biosynthesis I, IL-8, CXCR4, Neurotrophin/TRK, were differentially expressed in E17 fetal versus adult wounds. ${ }^{39}$

\section{Graphene and Graphene-Based Nanomaterials}

\section{Structure and Synthesis}

Graphene is an allotrope of carbon. Briefly, it is a twodimensional hexagonal lattice with a flat monolayer of carbon atoms formed by strong triangular bonds in $\mathrm{sp} 2$ hybrid orbitals, forming the basis of both 3D graphite and 1D carbon nanotubes. ${ }^{40,41}$ Graphene oxide (GO) is obtained by multi-step oxidation of graphene and ultrasonic purification. It contains hydroxyl, epoxy and carboxyl groups on the edge of the sheet, effectively increasing the interlayer spacing. ${ }^{42}$ Reduced graphene oxide (rGO) is produced from GO through a variety of reduction processes. ${ }^{43}$ To fully exploit the properties of graphene and its derivatives for biomedicine applications, it requires an ideal method for the mass production of this remarkable material. ${ }^{44}$ This remains a major challenge. So far, a number of techniques have been developed including mechanical cleavage, liquid-phase exfoliation and chemical vapor deposition, etc.

The graphite is composed of graphene sheets held together by Van der Waals interactions, therefore, graphene sheets can be torn down from graphite by applying mechanical force. ${ }^{45}$ Meyer et al prepared monolayer graphene flakes on top of an oxidized silicon wafer by mechanical cleavage. ${ }^{46}$ Analogously, liquid-phase exfoliation is defined that the graphite is dispersed and exfoliated in organic solvents. ${ }^{47}$ For the solvent-graphene interaction, the surface energies of the solvent match that of graphene, so that it could balance the energy to exfoliate graphene. ${ }^{48}$ Lange et al demonstrated that the scale-up of exfoliation could be achieved by shear exfoliation, ball milling or microfluidization. $^{49}$

Compared to the above, the oxidation-reduction of graphite is a more practical process for bulk-scale graphene materials. GO is generally fabricated by oxidizing graphite with a strong acid, mainly including Brodie, Staudenmaier, and Hummers method. ${ }^{50}$ Hummers is the most commonly 
used one at present because it is relatively time-efficient and safe. ${ }^{51}$ However, a conspicuous disadvantage of oxidationreduction methods is that the products are generally multilayer graphene or graphite microcrystals with different numbers of sheets, instead of monolayer nanosheets. Chemical vapor deposition is typically employed to fabricate monolayer graphene films on transition metals. ${ }^{52,53}$ Hydrocarbon gas (such as methane, ethane, or propane) is introduced into the reaction chamber as a carbon source. Graphene could be synthesized in this method with very few metallic residuals. $^{54}$ Tracy et al reported a novel method for lowtemperature synthesis of monolayer graphene at $450^{\circ} \mathrm{C}$ on a polycrystalline bimetal $\mathrm{Ni}$-Au catalyst. The electron beam co-deposition of the bimetal catalyst is the key procedure that enables the elimination of the pre-growth hightemperature annealing of the catalyst. ${ }^{55}$

\section{Biological Properties of GBNs}

The unique biological properties of GBNs determine their wide application in the health care industry. Here, we mainly emphasize the properties of graphene-based materials relevant to tissue engineering. The most prominent feature of graphene-based materials is their high specific surface area; this enables them to be loaded or to interact with not only inorganic molecules but also bioactive cells and vesicles. The morphology of GBNs could be observed by scanning electron microscopy (SEM) and transmission electron microscopy (TEM), manifested as randomly aggregated, flake-like sheets with folds on the porous surface, with or without face-to-face stacking of sheets ${ }^{43}$ (Figure 2). Lee et al measured the mechanical strength of monolayer graphene membranes by atomic force microscope (AFM) nanoindentation. ${ }^{56}$ In graphene-based composites, the mechanical nature of other soft materials can be significantly improved by graphene. ${ }^{57,58}$ The oxidation process damages the structure and mechanical properties of GO and other derivatives to some extent, nevertheless, both of them also appeal to researchers. ${ }^{59}$ The GO allowed the sustained release of N-acetyl cysteine (NAC), known as a clinically applied antioxidant to reduce the reactive oxygen species (ROS). The hybrid membrane incorporating NAC, collagen I, and GO exhibited more excellent mechanical properties and water retention capacity compared to collagen-only scaffold. ${ }^{60}$ Likewise, GO improves the mechanical properties of collagen membranes, as well as the roughness of the membrane surface slightly. These
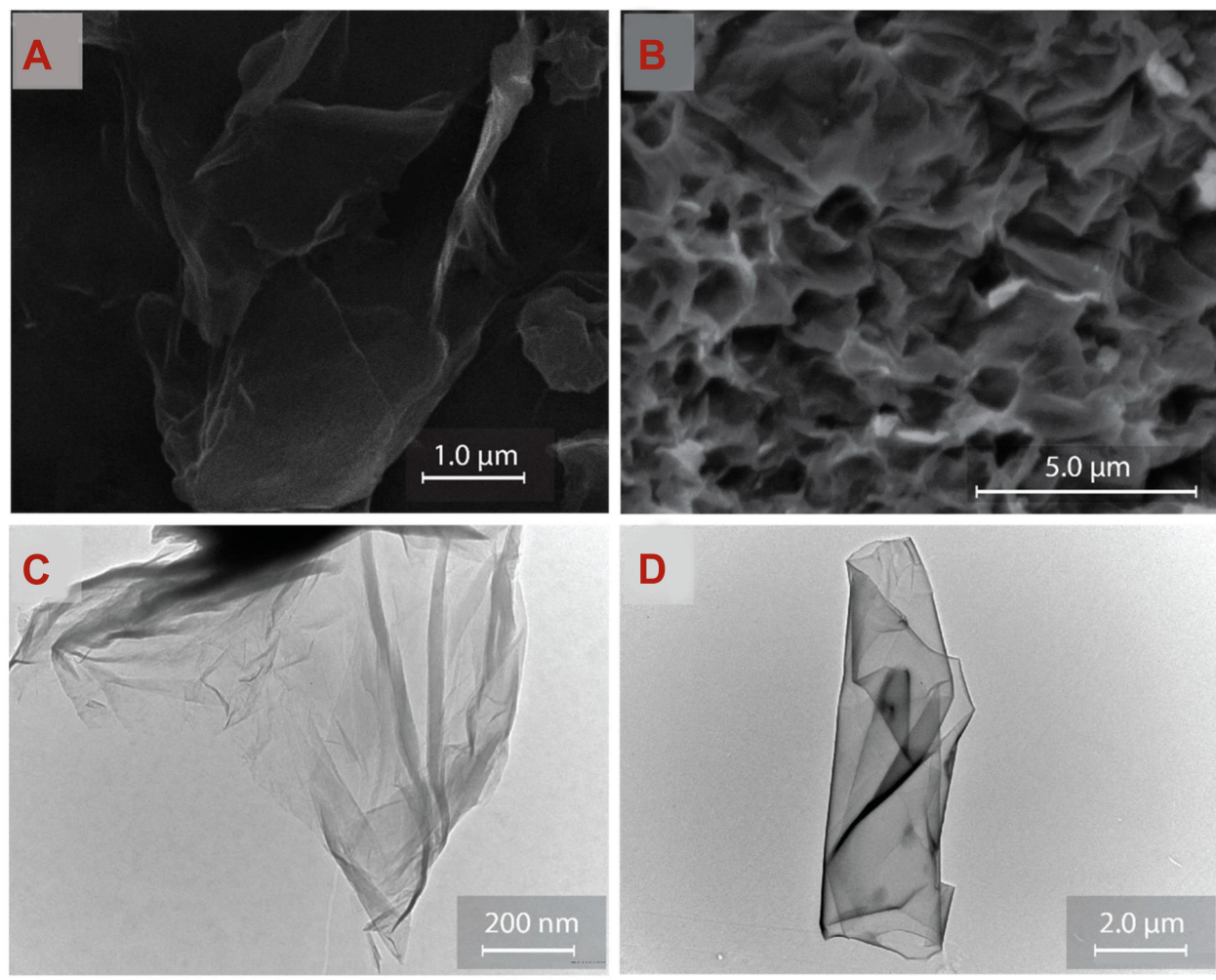

Figure 2 SEM morphology of (A) GO and (B) rGO. TEM images of (C) GO and (D) rGO.

Notes: Reprinted from Talia Tene, Gabriela Tubon Usca, Marco Guevara et al. Toward Large-Scale Production of Oxidized Graphene. Nanomaterials (Basel). 2020; I0(2):279. Copyright 2020, with permission from MDPI. ${ }^{43}$ 
changes in performance have been detected as beneficial to cell proliferation and adhesion. ${ }^{61}$ Aerogels made of natural polymer materials have been widely employed in artificial bone transplantation because of their high porosity and great biocompatibility. But due to their poor mechanical properties, natural polymer aerogels appeared to be unqualified for large bone defects repair. Compositing of GO for preparation of Col I aerogels enhanced the compressive modulus dose-dependently, so that more appropriate mechanical properties were achieved. ${ }^{62}$

The hydrophobic and electrostatic interaction could potentially increase the binding of graphene to other substances and improve several biological effects. The alkyl chains of graphene induce a high antiviral activity by secondary hydrophobic interactions. A perfect synergy between electrostatic and hydrophobic interactions induces a higher antiviral activity of graphene sheets. ${ }^{63}$ Similarly, by fine-tuning of molecular interaction between peptide nanofibers and graphene, hybrid hydrogels with tailored properties can be designed. ${ }^{64}$ However, even though the hydrophobic nature of graphene contributes to absorbing various hydrophobic organic molecules or polymers via van der Waals interaction, in terms of graphene, due to the hydrophobic nature, there arises a serious problem of agglomerations formation. In the last few years, studied revealed that GO may be an appropriate alternative for unique physicochemical properties, such as wide surface area, hydrophilia, flexibility, excellent electrical and thermal conductivity, and biocompatibility. ${ }^{65}$ In particular, oxygen-containing functional groups on the surface result in hydrophilic nature, allowing dispersibility, ${ }^{66}$ functional chemical modification and loading therapeutic components through both noncovalent interactions and covalent bonds via chemical reactions.

The excellent properties of GBNs for pathogens binding and inhibition have recently attracted much attention. The electrostatic interaction is the main driving force for binding graphene sheets to herpes simplex virus type 1 (HSV-1), ${ }^{63}$ while the antibacterial activity of graphene sheets mainly depends on the collision between sharp edges and bacteria, that is, "nano-knives" mechanism. ${ }^{67}$ Kim et al ${ }^{68}$ have prepared an effective antibacterial surface consisting of GO and molybdenum disulfide (MoS). It exhibits a promising antimicrobial property toward the Gram-negative bacteria Escherichia coli. The graphene derivatives-containing scaffolds are also employed to kill the captured bacteria by IR-laser irradiation of bacteriagraphene complex due to thermal IR-absorption properties of graphene, ${ }^{69}$ and this is a typical example for GBNs as photothermal therapy reagent (Figure 3). In addition, GBNs display a prominent ability of heat conduction ${ }^{70}$ and charge transport, ${ }^{71}$ inducing definite effects on target cells and tissues in biomedical application.

\section{Biocompatibility of GBNs Cytocompatibility and Cytotoxicity}

The cytocompatibility of GBNs with several cell lineages has been confirmed in recent years. Pristine graphene is found to promote adhesion and proliferation of murine fibroblasts L929 cell line within $24 \mathrm{~h}$ of culture, and seemingly no cytotoxicity appeared. Migration of cells on the surface of graphene substrate acts better in comparison to the uncoated glass surface. ${ }^{72}$ By introducing hydrophilic groups (nitrogen ions) into 3D graphene, L929 cells are detected in better growth condition. ${ }^{73}$ In bone tissue engineering, calcium silicate/graphene composites have been considered cell-friendly, and especially with a higher content of graphene were more favorable to the proliferation of human marrow mesenchymal stem cells. ${ }^{74}$ For peptide hydrogels, the addition of GO and rGO has no impact on the overall cytotoxicity to 3D-cultured hMSCs at $\mathrm{pH} 6$ over 14 days. ${ }^{64}$ On a 3D printed nanocomposite hydrogel filled with GO, the interaction of GO with the polymer network by hydrogen bonds allows a slow release of GO to the culture medium. hADSCs adhered to the scaffolds survive and distribute orderly, regardless of the formulation of the ink. ${ }^{75}$ In short, various factors like fabrication processes, dispersion in the medium, interacted functional groups all participate in the cells-GBNs performance. ${ }^{76,77}$

However, the potential toxicity triggers a series of safety concerns in the biomedicine application of GBNs, which is essential for the clinical translation. Wang et $\mathrm{al}^{78}$ found a dose-dependent relationship between $\mathrm{GO}$ and its cytotoxicity on human fibroblasts. When the GO concentration is less than $20 \mu \mathrm{g} / \mathrm{mL}$, GO is nearly non-toxic to fibroblasts, but when the GO concentration is greater than $50 \mu \mathrm{g} / \mathrm{mL}, \mathrm{GO}$ shows obvious cytotoxicity to fibroblasts. It mainly includes reducing cell adhesion and inducing apoptosis. It seems that GBNs induce a cytotoxic effect through a sustained mitochondrial depolarization. The increased ROS production mediates the depolarization, following the activation of $\mathrm{NADH}$ dehydrogenase and xanthine oxidase. $^{79}$ Analogously, though fluorinated graphene could improve cell adhesion on its surface in the early period, the intracellular ROS level is enhanced and the viability of MSCs declined after a longer incubation 


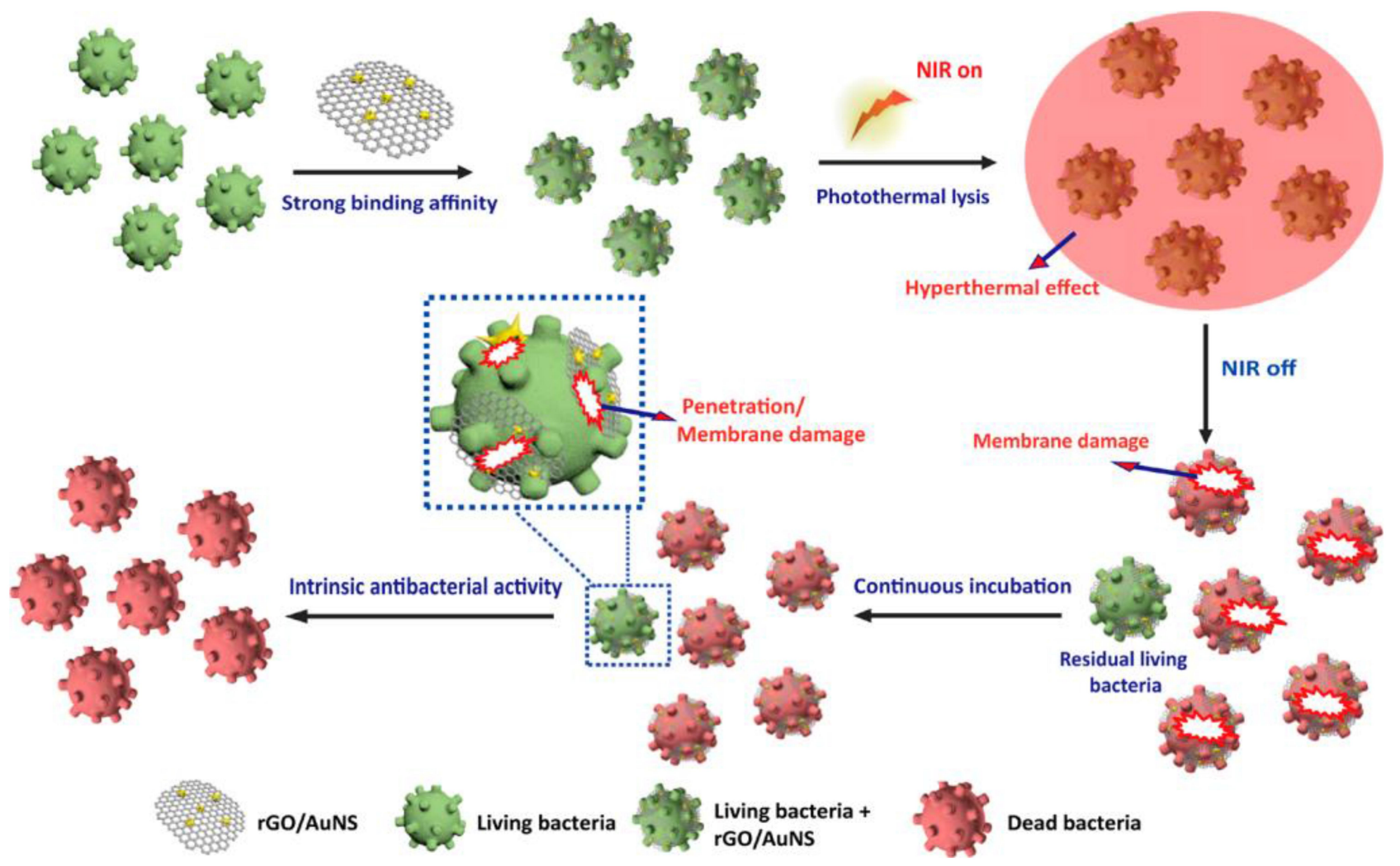

Figure 3 Schematic illustration of rGO/AuNS triggering intrinsic sterilization and antibacterial photothermal lysis.

Notes: Reprinted from Yonghai Feng, Qingyu Chen, Qing Yin et al. Reduced Graphene Oxide Functionalized with Gold Nanostar Nanocomposites for Synergistically Killing Bacteria through Intrinsic Antimicrobial Activity and Photothermal Ablation. ACS Applied Bio Materials. 2019; 2(2):747-756. Copyright 2019, with permission from American Chemical Society. ${ }^{150}$

period. ${ }^{80}$ Numerous functionalization methods are available to alleviate the cytotoxic response of graphene nanomaterials. ${ }^{81}$ GBNs coated with chitosan polymer exhibit reduced cytotoxicity compared to non-coated GBNs, which is reflected in fewer cell-cycle defects and higher cell viability. ${ }^{82}$

\section{Interactions in Biological Systems}

Once introduced into the systemic circulation, GBNs interact with the cells and biomolecules of the circulatory system, so hemocompatibility is a crucial index to evaluate the invivo compatibility. Geng et al have found that the red blood cells inoculated on fluorinated graphene exhibit no obvious hemolysis phenomenon, and the morphologies of platelets reveal that the pseudopods outstretch well on the surface. ${ }^{80}$ When the graphene quantum dots are injected (i.o) in mice, the uptake displays an age-related trend. It is, there is a decrease in the uptake of graphene quantum dots in aged mice when compared to young mice. ${ }^{83}$ To assess the response of the human epidermis to graphene exposure more realistically, instead of interfered with protein corona formation in the cell culture medium, a non-animal test has been implemented using the SkinEthic ${ }^{\mathrm{TM}}$ Reconstructed human Epidermis (RhE). The results confirm that the surfactants used to prepare GBNS, such as sodium dodecyl sulfate (SDS), are the initiators of the irritation, rather than the graphene material itself because the material is further washed by exfoliation is assessed non-irritant. ${ }^{84}$

Duch et $\mathrm{al}^{85}$ have found that the dispersion of GBNs and oxidation of the carbon backbone mattered in pulmonary toxicity. GO administered directly to the lungs of mice could cause severe and persistent lung injury with alveolar exudates and hyaline membrane formation. By contrast, inflammatory findings can be minimized for the intratracheally administration of highly dispersed nanoscale graphene. This difference is mainly due to the distinct levels of macrophage apoptosis. After intraperitoneal injection of GO nanoplatelets into adult male Wistar rats, a systematic toxicity appears. ${ }^{86}$ Histopathological analysis reveals dosedependent lesions in the liver, kidney, spleen, lung, intestine, and brain 21 days after GO nanoplatelets application. A low dose (50 or $150 \mathrm{mg} / \mathrm{kg}$ ) of GO does not exhibit body weight 
change, but a high dose $(500 \mathrm{mg} / \mathrm{kg})$ does. It has been demonstrated that the stable functionalization of GBNs could improve their in vivo pharmacokinetics and biodegradation, as well as minimize the potential toxicity. ${ }^{87}$ For instance, by conjugating polyethylene glycol (PEG) to GO via a cleavable disulfide bond, a composite product with negligible toxicity and ideal degradability can be obtained. ${ }^{88}$

\section{Application and Putative Mechanisms in Cutaneous Repairment}

Applications of GBNs as antibacterial agents have been reported, ${ }^{89}$ and there are immense synergistic actions with empirical antibiotics. For instance, pristine GO could disrupt the bacterial membrane, convenient for the bound antibiotics to inactivate bacteria. Coupled with the minimal toxicity to keratinocytes when the GO concentration is $<100 \mu \mathrm{g} / \mathrm{mL}$, it suggests a broad prospect for application in infected wounds. ${ }^{90}$ Ultrasonication could increase the dispersion and stability of the GO suspension, in the meanwhile, remove part of the oxygen-containing functional groups on the surface of nanosheets. In a rat excisional skin defect model, the wound healing rate of $1 \% \mathrm{UGO}$ group is even about the same as the group of basic fibroblast growth factor (bFGF) ${ }^{91}$

In recent years, the ease of modification and functionalization, as well as fabrication of composites enable the utilization of biocompatible GBNs in cutaneous repairment. ${ }^{81}$ Tissue engineering utilizes different GBNs-incorporated composite scaffold materials to provide a suitable microenvironment for cellular behavior (Figures 4 and 5). To enhance the healing efficacy via promoting multiple wound healing stages in sequence, the ceria-graphene nanocomposites have been designed. ${ }^{92}$ In detail, the semiconductor ceria nanoparticles can inactivate bacteria by ROS generation under white light irradiation at the inflammation stage. When stepping into the proliferation stage, the ceria and graphene nanoparticles would be separated. The former enter fibroblasts and scarify intracellular ROS, and the latter could act as a scaffold for fibroblasts migration. Thangavel et $\mathrm{al}^{93}$ developed the rGO loaded isabgol nanocomposite scaffolds (Isab $+\mathrm{rGO}$ ) to improve wound healing in normal and diabetic rats. 2, 2-diphenyl-2-picrylhydrazyl (DPPH) scavenging assay revealed a prominent feature of antioxidation, which could minimize the ROS generation and lipid peroxidation. The natural polyphenolic compound curcumin has been reported for its wound healing properties including antioxidation, anti-inflammatory, reepithelization, and tissue remodeling. The scaffold incorporating $\mathrm{rGO} / \mathrm{Ag}$ nanocomposites, curcumin or both of them has been prepared. ${ }^{94} \mathrm{rGO} /$ Ag nanocomposites increase the surface hydrophilicity of the scaffold, and therefore, the wettability is enhanced and cellular attachment on the scaffold is facilitated.

The capacity of absorbing excess exudates produced by the wounds is one of the typical advantages of hydrogels. Gelatinmethacryloyl (GelMA) based hydrogels can be swollen with around $1000 \%$ water during short-term immersion. ${ }^{95}$ The prepared GelMA hydrogel loaded with rGO showed angiogenic potential in chicken embryo angiogenesis (CEO) testing. Liang et $\mathrm{al}^{96}$ prepared a series of adhesive hydrogels for wound dressing based on hyaluronic acid (HA) -graftdopamine (DA) and rGO using an H2O2/HPR (horseradish peroxidase) system. Owing to excellent electrical conductivity, rGO improves the wound-healing effects when incorporated into HA-DA hydrogel dressing. By immunohistochemical staining, on the 7th and 14th days, the level of CD31 in the wounds treated with $\mathrm{HA}-\mathrm{DA} / \mathrm{rGO} 3$ was detected higher than that in the Tegaderm membrane group, illustrating an improving angiogenesis process induced by the composite hydrogel. Furthermore, the GO can be reduced by polydopamine (PDA) and endows the chitosan/silk fibroin scaffolds with good electrical conductivity. ${ }^{97}$ Generally, electroactive scaffolds as
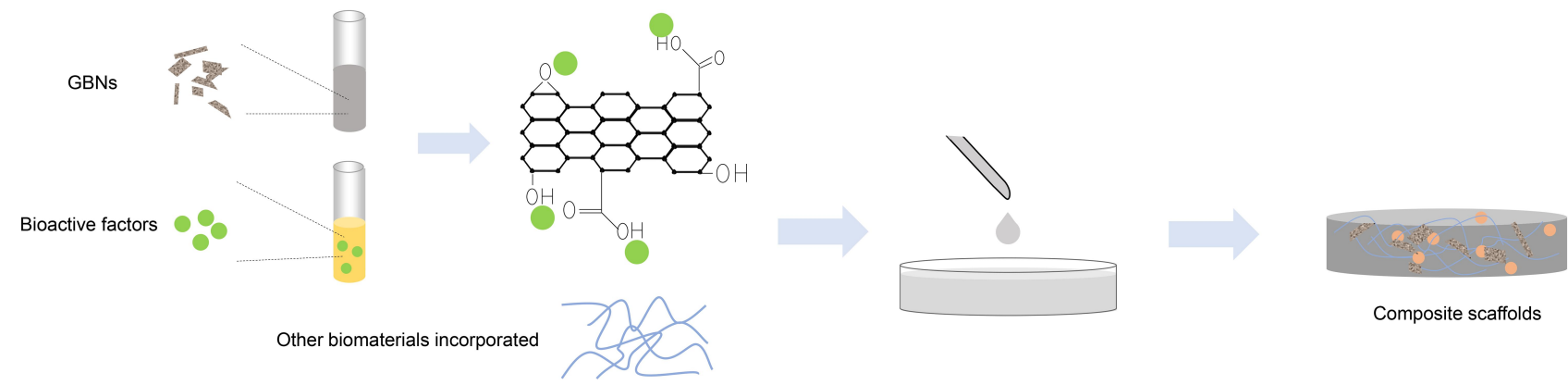

Figure 4 Schematic illustration of the common methods for preparing GBNs-incorporated composite scaffolds and wound dressings. GBNs could be combined with other biomaterials and drugs. In addition to enhancing the mechanical properties, GBNs could also improve the biological interactions by providing more anchoring sites for bioactive growth factors or specific drugs, utilizing the oxygen-containing functional groups of the basal plane and over the edges of the sheets. 


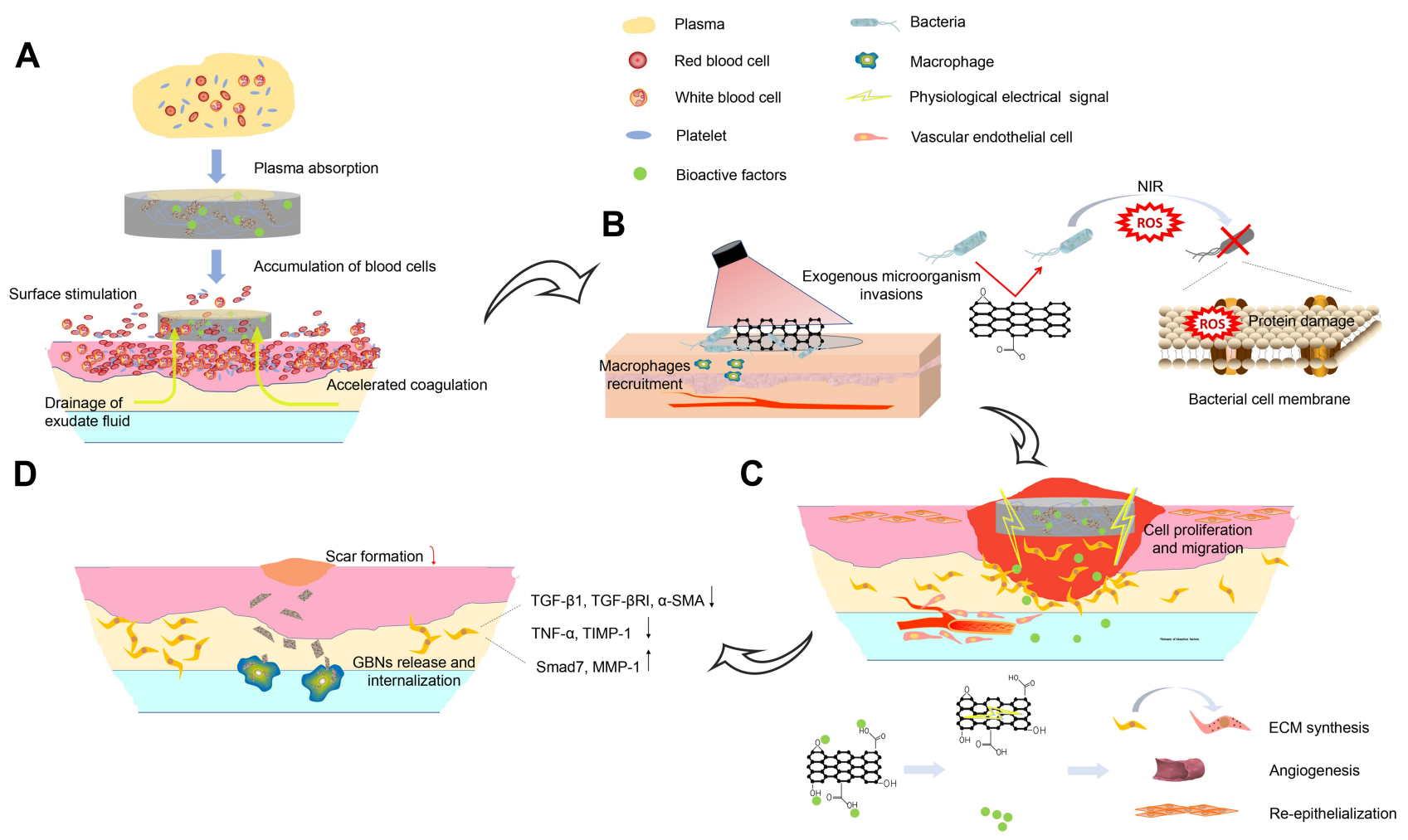

Figure 5 Schematic illustration of effect and putative mechanisms of GBNs on wound healing in multiple wound healing stages in sequence. (A) In the hemostasis phase, the high specific surface area enables nanofibers to absorb plasma fast. The oxygen polar groups can instantly stimulate erythrocytes and platelets at the interface, further promoting blood coagulation. (B) The composite scaffolds exhibit good antibacterial performance owing to the small pore size to inhibit microorganism invasions and the photothermal properties of GBNs. (C) Released bioactive factors and GBNs act synergistically on fibroblasts, keratinocytes and endotheliocytes et al during proliferation phase. (D) The presence of GBNs inhibits the fibroblasts from overactivation, accompanied by the down-regulation of scar-related genes. Highly expressed MMP in the extracellular matrix could facilitate the macrophage to internalize the GBNs through endocytosis, leading to the biodegradation.

described above could accelerate wound healing because they were able to respond to physiological electrical signals at wound sites during the healing process. Currently, most of the wound dressing hydrogels are chemically cross-linked, inducing a potential toxicity of residual organic cross-linkers. To avoid this, Yan et $\mathrm{al}^{98}$ designed an injectable hydrogel formed in situ by physical crosslinking. This PEP- Ag@rGO hydrogel could be administered by spraying the hybrid aqueous mixture onto the targeted skin area and transit immediately to the hydrogel in response to a temperature higher than $30^{\circ} \mathrm{C}$, thereby providing a stable dressing for the wound.

Aerogel is a gel material with gas as a dispersion medium. The solid phase of which is not only nano-scale but also porous. For instance, GO-COL aerogels ${ }^{62}$ have been observed a better repairing effect compared to aerogels prepared with Col only. Mellado et al ${ }^{99}$ generated dry and stable composite aerogels based on GO and poly (vinyl alcohol) (PVA) combined with natural extracts through the sol-gel method. This aerogel promotes hemostasis via reduction of the coagulation time during contact with whole blood. GO was likely to promote fibroblasts migration and proliferation when composited into collagen- $\mathrm{N}$-acetyl cysteine hybrid membrane. In the rat cutaneous wound model, there appeared a full healing on the 14th day after the application of the hybrid membrane which preceded the control group markedly. Furthermore, the GO-incorporated hybrid membrane noticeably down-regulated the scar-related gene expression, which revealed a potential effect to facilitate scar-free wound closure. ${ }^{60}$ By mimicking the hierarchical microstructure of nacre, GO-chitosan-calcium silicate film prepared by Xue et $\mathrm{al}^{100}$ has a "brick-and-mortar" layered nanostructure and orderly porous lamellar micron-scale structure, endowing the film not only good tensile strength but also desirable breathability and water absorption property.

\section{Application in Drugs Delivery and Cell Scaffolds}

GBNs could load drugs noncovalently via $\pi-\pi$ stacking interactions, hydrogen bonding, or hydrophobic interactions. The ability to cross cell membranes and the high specific surface area supply important advantages of 
GBNs for drug delivery, and related studies have been widely conducted. ${ }^{59}$ For instance, GO could efficiently deliver chlorogenic acid (CA) under phosphate buffer solution after forming a CAGO nanocomposite. Thermal analyses showed enhancement in the thermal stability of CAGO nanocomposite compared to the free drugs. ${ }^{101}$ The coating of GO on drug-delivery systems could also lead to a controlled release because of the intermolecular interactions $(\pi-\pi$ stacking, hydrogen-bonding or electrostatic retardation) between the diffusing drugs and the decorations. ${ }^{102}$ They possess a greater advantage of delivering drugs at the targeted site with ease at a higher rate. Moreover, GBNs can easily be monitored and, even imaged in vivo by some functionalization methods, for example, incorporating with superparamagnetic nanoparticles as carriers of therapeutic factors. ${ }^{103}$

GO exhibits a high drug loading capacity for aromatic molecules. It could release drugs and translocate into the nucleus slowly, which is pH-sensitive and initiated by NIR. ${ }^{104}$ A promising drug delivery system ought to avoid initial burst release and loss of bioactivity of drugs in in vitro and in vivo applications. ${ }^{105}$ The conjugation of PEG can weaken the bond between GBNs and drugs, which could induce a better drug release and treatment effect. Fourier transform-infrared spectroscopy (FT-IR) confirms that the $\pi-\pi$ stacking interaction mediates the binding of drugs to the surface of GBNs. ${ }^{106}$ Although the $\pi-\pi$ interactions are important in drug loading, the dominant mechanism is the electrostatic interaction of ionized drugs with GO (especially through H-bonding). Rasoulzadeh et al tested the loading and releasing behavior of DOX, indicating that the nanocomposite hydrogels have better drug loading properties in comparison to pure hydrogel. ${ }^{107}$ These nanocomposite materials usually exhibit excellent water dispersity and stability, ${ }^{108}$ which provides prospects for clinical applications.

The various physiochemical properties of graphene nanomaterials permit a favorable microenvironment for the enhanced growth of cells or thus provide required stimuli for cellular differentiation to specific cell lineage. $\mathrm{Wu}$ et $\mathrm{al}^{109}$ prepared starch nanofibers with incorporated GO by electrospinning. The viability of osteoblasts cell line MG 63 cultured on it remains unaltered in the case of appropriate GO concentration. GO promotes mineralization of calcium and phosphorus in a serum-like medium during the cell culture. The application of GO as a nanofiller into self-assembling peptide not only benefits for mimicking the mechanical properties of nucleus pulposus but also promotes high viability and remains a metabolic activity of bovine nucleus pulposus cells over the 7 days of culture. ${ }^{110}$

GBNs also show the potential to fabricate alternative tools that can substantially affect gene expression of locally resident cells and change the tissue microenvironment. ${ }^{111}$ Graphene could act as a biocompatible and conductive substrate for human induced pluripotent stem cells (hiPSCs) and mimic the biomimetic conductive cardiogenic niche to promote the self-renewal and cardiac differentiation of hiPSCs. ${ }^{112,113}$ It is reported that highly conductive GBNs, such as the carbon nanotubes, could enhance the neuronal differentiation of multipotent autologous cells in most cases. On the contrary, several GBNs-conjugated composite scaffolds which are less conductive appeared to boost the expression of myogenic-lineage marker genes. ${ }^{113}$ Saravanabhavan et al developed a GO functionalized chitosan nanoparticle to carry osteosarcoma cells-targeted siRNA. It shows a controlled $\mathrm{pH}$-related release so that it facilitates the targeting of acidic tumor site ${ }^{114,115}$ (Table 1).

\section{MSC and MSC-Exos}

\section{MSCs in Cutaneous Wound Healing and Joint Application with GBNs}

In the field of regenerative medicine, mesenchymal stem cells (MSCs) have been identified as a critical element for outstanding capacities including proliferation, multipotent differentiation, and bioactive paracrine factors release. MSCs derived from various tissues, such as bone marrow, subcutaneous adipose tissue and umbilical cord, are demonstrated a therapeutic potential for wound healing disorders. Previously, MSCs are usually implanted as a cell therapy by intravenous administration or subcutaneous injection around the wound. ${ }^{116}$ In fact, the healing effect is dependent on not only the localization of implanted MSCs but also their survival rate within the wound site and the induced microenvironment change. The features of high cell yields and being simply obtainable make MSCs advantageous to be utilized in tissueengineering scaffolds. Based on materials science, many kinds of nanomaterials have emerged and been developed as biomimetic models to optimize the stem cell functions. ${ }^{87}$

Due to the interactions between graphene and stem cells, extensive research has been devoted to developing GBNs that are capable of imitating the physiological 
Table I A List of Different Types of GBNs Used in Biomedical Applications

\begin{tabular}{|c|c|c|c|}
\hline GBNs & $\begin{array}{l}\text { Type of Cells or } \\
\text { Tissue }\end{array}$ & Effect & Ref. \\
\hline $\begin{array}{l}\text { CS/graphene } \\
\text { composites }\end{array}$ & $\begin{array}{l}\text { Human marrow stem } \\
\text { cells }\end{array}$ & $\begin{array}{l}\text { Cell-friendly, and higher contents of graphene were more favorable to the } \\
\text { proliferation of cells. }\end{array}$ & [74] \\
\hline GO and rGO hydrogel & hADSCs & hADSCs adhered on the scaffolds survived and distributed orderly. & [75] \\
\hline GO suspension & $\begin{array}{l}\text { Rat excisional skin } \\
\text { defect }\end{array}$ & $\begin{array}{l}\text { One of GO concentration group even exhibited about the same wound healing rate } \\
\qquad \text { as the bFGF. }\end{array}$ & [9I] \\
\hline $\begin{array}{l}\text { Ceria-graphene } \\
\text { nanocomposites }\end{array}$ & Dermal fibroblasts & $\begin{array}{l}\text { The ceria entered fibroblasts scarifying intracellular ROS, and graphene could act as } \\
\qquad \text { a scaffold for fibroblasts migration. }\end{array}$ & [92] \\
\hline PEGylated nano GO & HeLa cells & $\begin{array}{l}\text { Release drugs and translocate into the nucleus slowly, and the nucleus translocation } \\
\qquad \text { was NIR initiated and } \mathrm{pH} \text { sensitive. }\end{array}$ & {$[104]$} \\
\hline $\begin{array}{l}\text { GO-incorporated } \\
\text { starch nanofibers }\end{array}$ & $\begin{array}{l}\text { Osteoblasts cell (MG } \\
63)\end{array}$ & GO promoted mineralization of calcium and phosphorus & {$[109]$} \\
\hline $\begin{array}{l}\text { GO- incorporated self- } \\
\text { assembling peptide }\end{array}$ & $\begin{array}{l}\text { Bovine nucleus } \\
\text { pulposus cells }\end{array}$ & $\begin{array}{l}\text { Cellular viability and metabolic activity were promoted over the } 7 \text { days of culture, } \\
\text { which benefiting for mimicking the mechanical properties of nucleus pulposus, }\end{array}$ & {$[110]$} \\
\hline Graphene & hiPSCs & $\begin{array}{l}\text { Graphene mimicked the biomimetic conductive cardiogenic niche and promoted self- } \\
\qquad \text { renewal and cardiac differentiation of hiPSCs. }\end{array}$ & {$[112]$} \\
\hline $\begin{array}{l}\text { PLLA and rGO, CNT, } \\
\text { CNHs }\end{array}$ & hCMCs & $\begin{array}{l}\text { Highly conductive CNTs boost neuronal differentiation, while less conductive CNH, } \\
\text { RGO@PLLA, and PLLA scaffolds enhance the expression of myogenic markers. }\end{array}$ & [113] \\
\hline $\begin{array}{l}\text { PEG diamine/R8- } \\
\text { functionalized GO }\end{array}$ & Breast cancer cell & $\begin{array}{l}\text { The effective uptake of the nanocarrier by the cells shows superior cytocompatibility, } \\
\text { and protects the siRNA and pDNA against enzyme degradation. }\end{array}$ & {$[115]$} \\
\hline $\begin{array}{l}\text { GO functionalized } \\
\text { chitosan nanoparticle }\end{array}$ & $\begin{array}{l}\text { Osteosarcoma cells } \\
\text { (Saos-2 and MG-63) }\end{array}$ & $\begin{array}{c}\text { The osteosarcoma cells-targeted siRNA loaded by composite nanoparticles were } \\
\text { released in a controlled fashion at acidic pH }\end{array}$ & [114] \\
\hline
\end{tabular}

Abbreviations: CS, calcium silicate; hADSCs, human adipose-derived stem cells; bFGF, basic fibroblast growth factor; ROS, reactive oxygen species; NIR, near-infrared ray; PLLA, poly-L-lactic acid; CNH, carbon nanohorn; hCMCs, human circulating multipotent stem cells.

microenvironment of MSCs and controlling their fate. $^{72,117-119}$ In diabetic wound repair, nanomaterial-drugcollagen hybrid scaffolds have been fabricated based on the PEGylated GO and acellular dermal matrix (ADM). Excellent cytocompatibility for GFP-labeled MSCs, as well as the induced adipogenic and osteogenic differentiation, is detected by two-photon excitation fluorescence and second-harmonic generation (TPEF-SHG) microscopy $^{120}$ (Figure 6). The combined application of growth factors or other active molecules also broadens the prospects of the GBNs with MSCs. Incorporation of GO into hybrid hydrogel prolonged the in vitro TGF- $\beta 3$ retention for up to 4 weeks. ${ }^{121}$ However, cell transplantation of MSCs is still associated with problems including safety concerns for potential tumorigenicity and immunological rejection. The exosomes derived from MSCs have the potential to avoid this and, meanwhile, maintain the paracrine secretome of the specific MSCs.

\section{MSC-Exos: An Emerging Cell-Free} Approach to Cutaneous Wound Repair

MSC-Exos are considered to be one of the most important secreted products of MSCs. Due to its high stability, nonimmune rejection, and easy-to-control concentration, it could mediate intercellular communication and enhance wound healing. ${ }^{122}$

Li et $\mathrm{al}^{123}$ studied the effect of human umbilical cord mesenchymal stem cells (hUMSCs) on the inflammatory response in diabetic rat burn models. They have found that the endogenous miR-181c of hUMSC-Exos can inhibit Tolllike Receptor 4 signaling pathway, thereby attenuating the lipopolysaccharide-mediated inflammation. After administration of hUMSC-Exos overexpressing miR-181c, the expression of inflammatory factors such as TNF- $\alpha$ and IL$1 \beta$ is up-regulated, while the anti-inflammatory factor IL-10 is down-regulated. It is found that Exosomes derived from adipose-derived stem cells (ADSC-Exos) may be taken up 
A
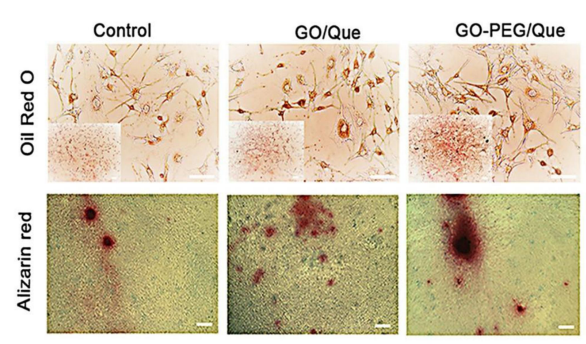

C
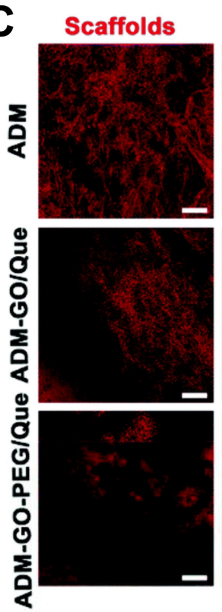

MSCs
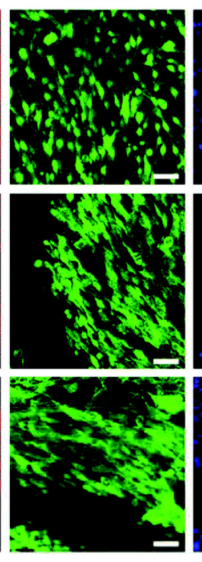

DAPI
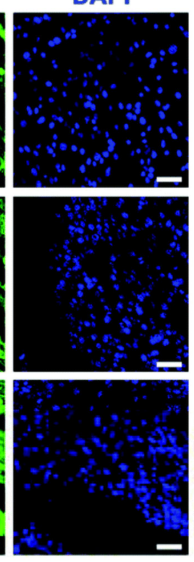

B
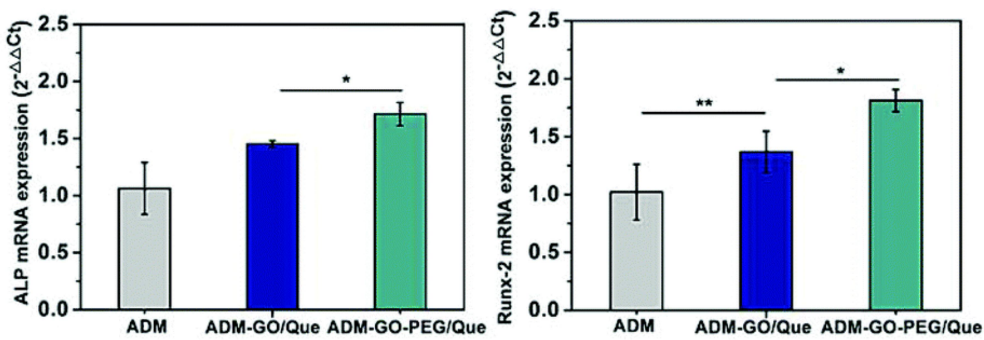

Merge
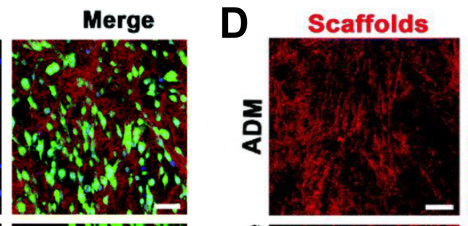

MSCs
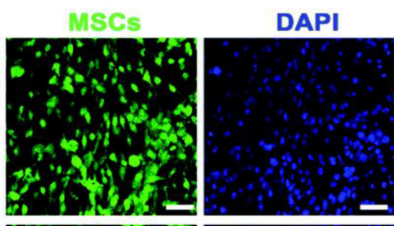

Merge

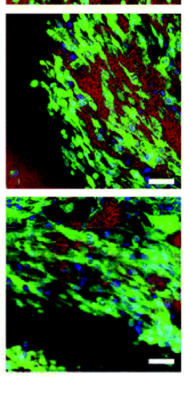

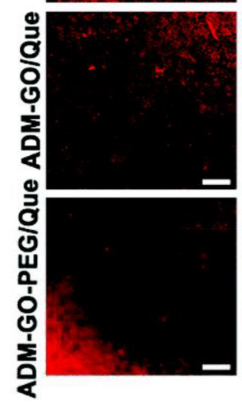
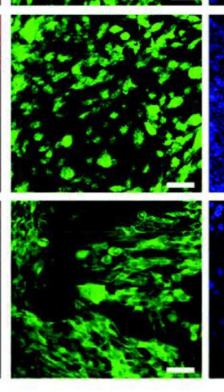

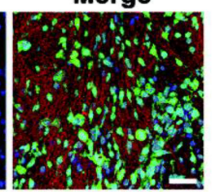

Figure 6 (A) Positive oil red O staining of MSCs after incubation with GO/Que or GO-PEG/Que indicates their potential to differentiate into adipocytes. (B) Quantitative analysis of the mRNA expression of ALP and Runx-2 of MSCs seeded on the ADM, ADM-GO/Que, and ADM-GO-PEG/Que scaffolds (*p $\leq 0.05$, **p $\leq 0.0 \mathrm{I}, \mathrm{n}=3$ ). TPEFSHG imaging showed that the PEGylated GO exhibited improved (C) osteogenic and (D) adipogenic differentiation of MSCs on the scaffolds. Scale bar: 50 m.

Notes: Reprinted from Jing Chu, Panpan Shi, Wenxia Yan et al. PEGylated graphene oxide-mediated quercetin-modified collagen hybrid scaffold for enhancement of MSCs differentiation potential and diabetic wound healing. Nanoscale. 2018;10(20):9547-9560. Copyright 2018, with permission from The Royal Society of Chemistry. ${ }^{120}$

and internalized by dermal fibroblasts to stimulate migration, proliferation and collagen synthesis in a dose-dependent manner with increased expression of N-cadherin, cyclin-1, PCNA and collagen I, III ${ }^{124}$ (Figure 7). Our group has demonstrated that the ADSC-Exos could act on the dermal fibroblasts in vitro and in vivo via PI3K/Akt signaling pathway, so that optimize the collagen deposition and wound closure. ${ }^{125}$ Nonetheless, hUMSCs-Exos inhibits the proliferation and migration of fibroblasts to prevent excessive tissue proliferation under high cell density. ${ }^{126}$ The hUMSCsExos-derived protein 14-3-3 $\zeta$ contributes to this effect by promoting the phosphorylation of protein YAP and activating the Hippo-YAP pathway, thereby having a reverse effect on the $\mathrm{Wnt} / \beta$-catenin signaling pathway.

Accessibility requirements for the source of exosome isolation spawn the application of massproduced sample from the human body. The menstrual blood-derived mesenchymal stem cells (MenSC)derived exosomes could resolve inflammation via induced M1-M2 macrophage polarization. ${ }^{127}$ Exosomes derived from MSCs in human urine samples
(USC-Exos) have been demonstrated the ability of promoting angiogenesis of diabetic wounds, and its advantage lies in the simple, safe, non-invasive and low-cost isolation procedure. ${ }^{128}$

Recent studies indicate that exosomes derived from modified or pretreated MSCs may exhibit a superior wound healing property. Ding et $\mathrm{al}^{129}$ isolated exosomes from BMSCs preconditioned by deferoxamine. The obtained exosomes could activate pathways pivotal in skin wound healing, such as AKT, extracellular signaling kinase (ERK), and transcriptional activator 3 (STAT3). Blue light illumination of hUMSCs could enhance the proangiogenic ability of their exosomes in tissue repair with an increase in miR-135b-5p and miR499a-3p expression. ${ }^{130}$ Exosomes derived from MSCs transfected with IncRNA H19 could suppress apoptosis and inflammation of fibroblasts in diabetic foot ulcer (DFU). It has been observed that the lncRNA H19 in MSCs is transferred to fibroblasts through exosomes and functions through the lncRNA H19/miR-152-3p/PTEN axis. $^{131}$ 


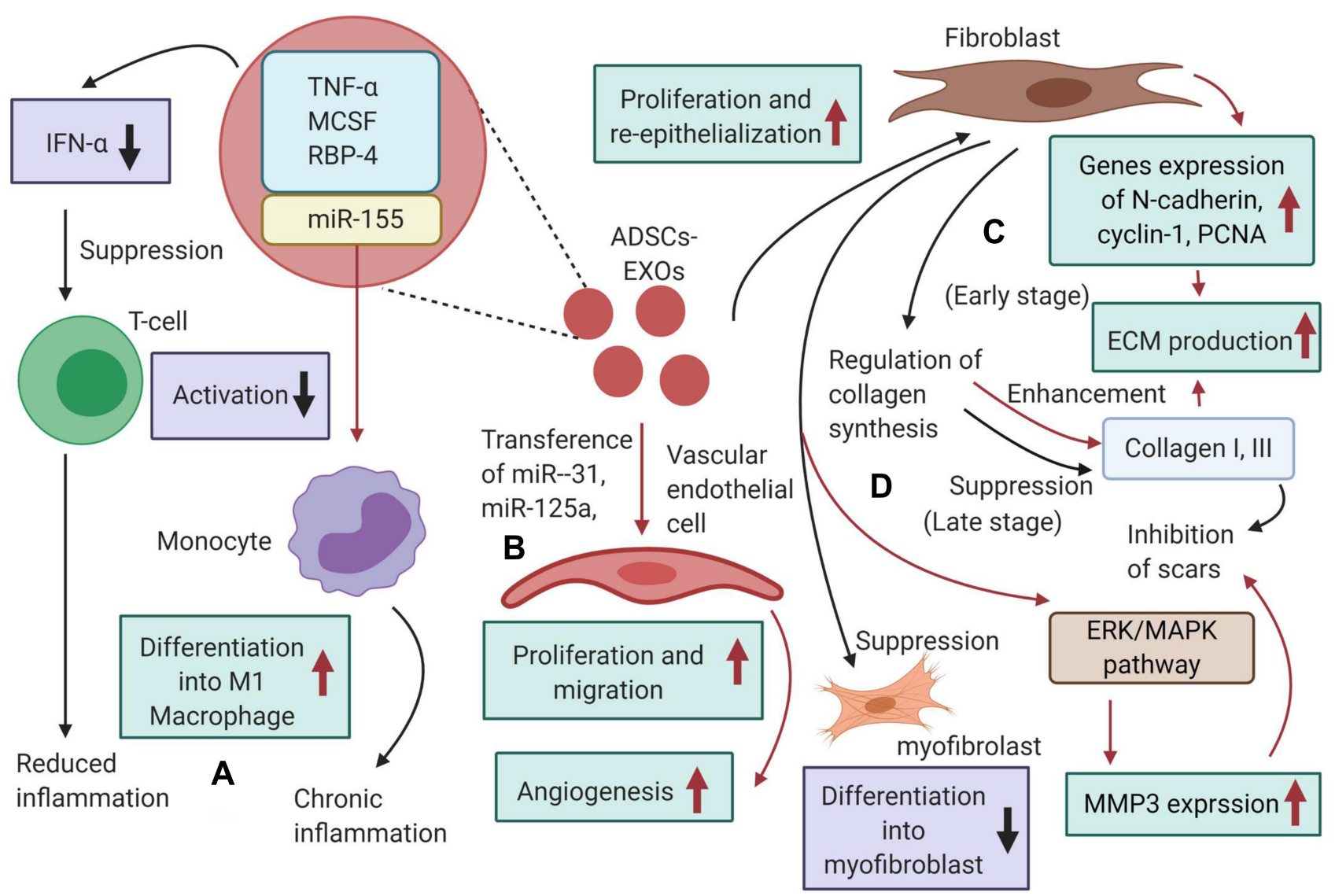

Figure 7 Mechanisms by which ADSCs-Exos could promote wound healing. (A) ADSCs-Exos contain immunoregulatory proteins and subsequently inhibit the activation of $\mathrm{T}$ cells, resulting in reduced inflammation. (B) ADSCs-Exos can transfer miRNA-I25a and miRNA-3I to vascular endothelial cells, promoting angiogenesis. (C) In the proliferation phase, ADSCs-Exos could stimulate N-cadherin, cyclin-I, PCNA and collagen I, III expression and increase ECM production; (D) in the matrix remodeling phase, ADSCs-Exos prevent the differentiation of fibroblasts into myofibroblasts, and reduce scarring and activate the ERK/MAPK pathway to increase MMP3 expression. Notes: Reprinted from Yang An, Shuyan Lin, Xiaojie Tan et al. Exosomes from adipose-derived stem cells and application to skin wound healing. Cell proliferation. 202I; el2993. Copyright 2021, with permission from John Wiley \& Sons Ltd. ${ }^{151}$

\section{Exploration of Novel Effective MSC-Exos Administration Routes for Wound Repair} In most of the above studies, exosomes are applied through subcutaneous injection to several sites around the wound. Together with other common methods of administration, like intravenous injection, they face the challenges of burst release and rapid clearance. Therefore, it is not conducive to play the due role during several complex stages in the whole wound repair process. At present, researchers are moving towards a new strategy based on loading MSCExos by patches, injectable microcarriers or hydrogels, aimed at maintaining the function of exosomes at the wound site and enhancing efficiency and safety.

As for carrier materials, the controlled release function is required. Chitosan and relevant compounds are ideal carriers for the sustained release of nanoparticles including exosomes. ${ }^{82,132,133}$ The delivery of exosomes derived from
miR-126-3p-overexpressing synovium mesenchymal stem cells is markedly prolonged to achieve a long-term exosome exposure to the wound site. With the increase of immersion time in the conditioned medium, the number of labeled exosomes in the perinuclear region of microvascular endothelial cells slowly increases. ${ }^{134}$ Shi et al prepared the chitosan/silk hydrogel sponge by freeze-drying method to be a scaffold for exosomes. Since chitosan is a hydrophilic polymer, this hydrogel sponge shows good swelling behavior, creates a moist environment and enhances the angiogenesis and neuronal ingrowth. ${ }^{135}$ Alginate-based hydrogels have been designed to encapsulate ADSC-Exos to fabricate a bioactive scaffold, ${ }^{136}$ which is tested biodegradable and biocompatible, reflecting potential as a cell-free therapy.

Several studies have shown that various artificial injectable hydrogels can not only promote the sustained 
release but also increase the local retention of exosomes in vivo. ${ }^{135-137}$ An injectable, self-healing and antibacterial polypeptide-based FHE hydrogel (F127/OHA-EPL) has been developed with a stimuli-responsive exosomes release for enhancing chronic wound healing and complete skin regeneration. ${ }^{138}$ The ureido-pyrimidinone supramolecular hydrogel undergoes a solution-to-gel transition when the $\mathrm{pH}$ is switched from high to neutral, with a threshold at $\mathrm{pH} \approx 8.5$. Moreover, nanoparticle tracking analysis (NTA) shows that the size distribution profile remains normal despite encapsulated and released from the hydrogel. ${ }^{137}$ Adipose tissue is abundant around the local cutaneous wound; thus, ADSC-Exos with bioactive constituents have earned boomed studies on exploring novel delivery systems for them. Likewise, adipose stromal cell-derived exosomes were released in a $\mathrm{pH}-$ dependent manner from injectable adhesive thermosensitive multifunctional dressing to promote angiogenesis in the diabetic wound. ${ }^{139} \mathrm{An}$ acellular tissue patch prepared from photoinduced imine crosslinking hydrogel glue, combined with stem cellderived exosomes, can be well integrated with the cartilage matrix and promote cell deposition at cartilage defect. The unique delivery by patch matches the intricate cartilage surface well, thus facilitating the contact of MSC-Exos with articular cartilage ${ }^{140}$ (Figure 8).

In general, the exosome-carrier compound displays better healing outcomes than the exosomes or carrier materials alone, suggesting a synergistic effect through the sustained release of MSC-Exos. Further exploration of the putative mechanism is required which is essential to realize the transformation of research results into reliable clinical applications.

\section{Outlook: Combined Application and Feasibility}

In recent years, the cutaneous wound repair, as a complex process involving different types of resident and recruited circulating cells, has been well studied. The single-cell technologies allow unraveling the heterogeneous of these cell subsets as well as identifying and characterizing novel rare cell subsets, instead of detecting the average cellular output of biology states. ${ }^{141}$ It is revolutionary in understanding the mechanisms of normal and impaired wound repair.

Various natural ${ }^{142}$ and synthetic ${ }^{143}$ materials loaded with Exos have been reported as cell-free therapeutic

C
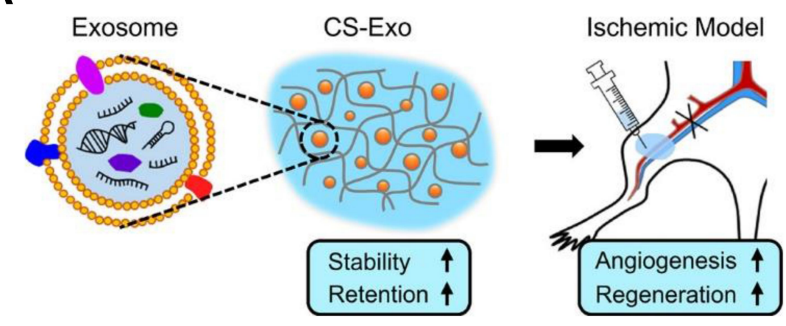

B

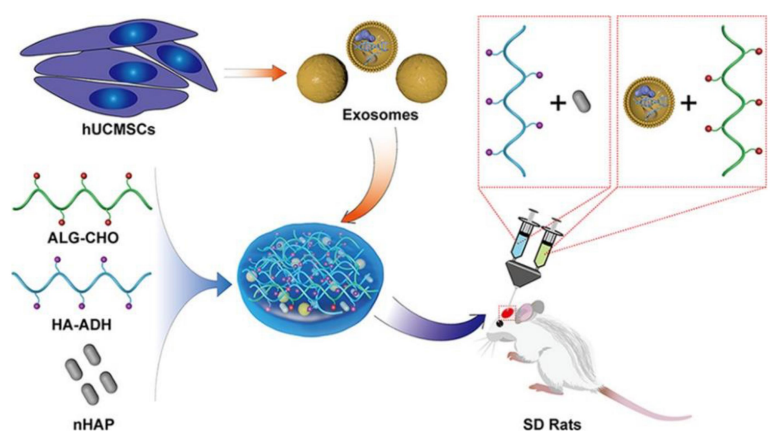

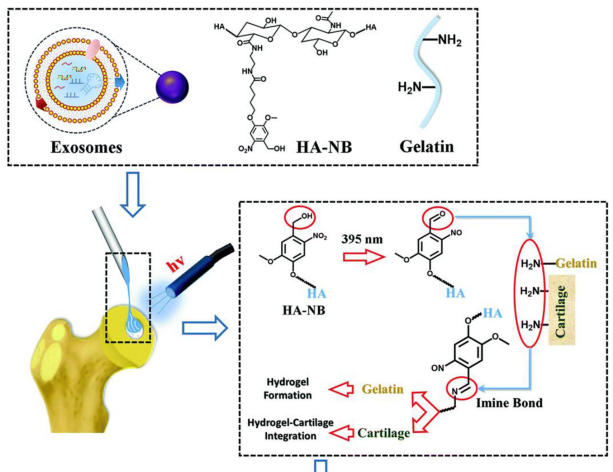

तु

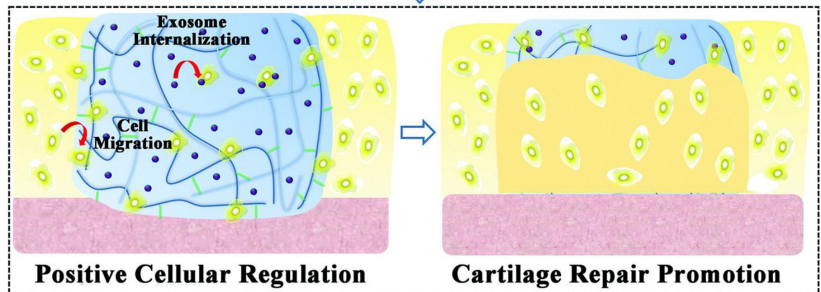

Figure 8 Typical schematic representations of MSC-Exos-incorporated hydrogels or scaffolds based on (A) chitosan, (B) sodium alginate, (C) hyaluronic acids for tissue regeneration.

Notes: (A)Reprinted from Kaiyue Zhang, Xiangnan Zhao, Xiaoniao Chen et al. Enhanced Therapeutic Effects of Mesenchymal Stem Cell-Derived Exosomes with an Injectable Hydrogel for Hindlimb Ischemia Treatment. ACS Appl Mater Interfaces. 2018;10(36):3008I-3009I. Copyright 20I8, with permission from ACS publication. ${ }^{132}$ (B) Reprinted from Shuo Yang, Biao Zhu, Peng Yin et al. Integration of Human Umbilical Cord Mesenchymal Stem Cells-Derived Exosomes with Hydroxyapatite-Embedded Hyaluronic Acid-Alginate Hydrogel for Bone Regeneration. ACS Biomater. Sci Eng. 2020;6(3):1590-1602. Copyright 2020, with permission from ACS publication. ${ }^{152}$ (C) Reprinted from Xiaolin Liu, Yunlong Yang, Yan Li et al. Integration of stem cell-derived exosomes with in situ hydrogel glue as a promising tissue patch for articular cartilage regeneration. Nanoscale. 2017;9(13):4430-4438. Copyright 2017, with permission from The Royal Society of Chemistry. ${ }^{140}$ 
applications for wound healing. This review proposes the joint application of GBNs and MSC-Exos, and strives to develop the GBNs as the delivery system of MSC-Exos. It matters whether the two can complement each other to achieve a good synergistic effect in wound repair. It is exciting to note that the high specific surface area and loading rate of GBNs would be helpful to increase the effective dose of MSC-Exos acting in local wound sites, as well as the strong sustained release ability allows MSCExos to obtain an enhanced lasting acting time, retention rate and stability. The interaction of GBNs with cells involved in wound healing response helps to increase the specificity of MSC-Exos to regulate gene expression. Coupled with the inherent antibacterial properties of GBNs, they get prospects to further improve the reepithelialization, angiogenesis and collagen maturation, as for exploiting novel skin substitutes and cell-free therapies.

Additionally, the consociation of GBNs with 3Dbioprinting is also worthy of attention, to obtain tailormade structures and functions for tissue engineering and 3D cell culture. ${ }^{144,145}$ Studies have revealed that the mixture of graphene into composite bio-inks helps to enhance the mechanical properties and printability. ${ }^{146}$ The aforementioned biological properties of GBNs provide additional advantages in cell-containing bio-ink such as increasing cellular vitality in the matrix. During in vitro or in vivo culture of printed products, the antioxidant activity could play a role in alleviating ROS generation and lipid peroxidation. ${ }^{95}$ The hydrophobic, hydrophilic, and $\pi-\pi$ interactions endow GBNs with the ability to adsorb proteins from biological surrounding to the $3 \mathrm{D}$ construct. ${ }^{75}$ The addition of GO could increase the cellular proliferation directionality along the printed threads, which offers a novel solution for the reconstruction of anisotropic structures, such as tendons and muscle fibers.

The bottlenecks faced include that the microcosmic mode and characteristics of contact between GBNs and MSC-Exo need to be explored in depth, so that to improve the attachment, release properties and compatibility with membrane structure. The balance between regenerative value and potent toxicity should be properly handled. ${ }^{147}$ Furthermore, unlike extensively studied tumor cells, the yield of MSC-Exosomes is a limiting factor for large-scale production for cell-free therapies. ${ }^{148}$ Therefore, it is of great necessity to increase the yield without reducing their therapeutic efficacy. ${ }^{149}$

\section{Disclosure}

The authors reported no conflicts of interest for this work.

\section{References}

1. Liao C, Li Y, Tjong SC. Graphene nanomaterials: synthesis, biocompatibility, and cytotoxicity. Int J Mol Sci. 2018;19 (11):3564. doi:10.3390/ijms19113564

2. Novoselov KS, Geim AK, Morozov SV, et al. Electric field effect in atomically thin carbon films. Science. 2004;306 (5696):666-669. doi:10.1126/science.1102896

3. Huang L, Santiago D, Loyselle P, Dai L. Graphene-based nanomaterials for flexible and wearable supercapacitors. Small. 2018;14(43):e1800879. doi:10.1002/smll.201800879

4. Singh E, Meyyappan M, Nalwa HS. Flexible graphene-based wearable gas and chemical sensors. ACS Appl Mater Interfaces. 2017;9(40):34544-34586. doi:10.1021/acsami.7b07063

5. Wang Z, Gao H, Zhang Q, Liu Y, Chen J, Guo Z. Recent advances in $3 \mathrm{D}$ graphene architectures and their composites for energy storage applications. Small. 2019;15(3):e1803858.

6. Bao Q, Loh KP. Graphene photonics, plasmonics, and broadband optoelectronic devices. ACS Nano. 2012;6(5):3677-3694. doi:10.1021/nn300989g

7. Li J, Zhang P, He H, et al. Enhanced thermal transport properties of epoxy resin thermal interface materials. ES Energy Environ. 2019;4:41-47.

8. Tang C, Yu X, Li G, Yang N, Lü J. Phonon thermal transport properties of graphene periodically embedded with four- and eight-membered rings: a molecular dynamics study. ES Mater Manuf. 2018;3:16-21.

9. Zhang Y, Yan Y, Guo J, et al. Superior thermal dissipation in graphene electronic device through novel heat path by electron-phonon coupling. ES Energy Environ. 2019;8:42-47.

10. Wu X, Liu R. Near-field radiative heat transfer between graphene covered biaxial hyperbolic materials. ES Energy Environ. 2020;10:66-72.

11. Cai J, Tian J, Gu H, Guo Z. Amino carbon nanotube modified reduced graphene oxide aerogel for oil/water separation. ES Mater Manuf. 2019;6:68-74.

12. Nidamanuri N, Li Y, Li Q, Dong M. Graphene and graphene oxide-based membranes for gas separation. Eng Sci. 2020;9:3-16.

13. Orsu P, Koyyada A. Recent progresses and challenges in graphene based nano materials for advanced therapeutical applications: a comprehensive review. Mater Today Commun. 2020;22:100823. doi:10.1016/j.mtcomm.2019.100823

14. Kim HS, Sun X, Lee JH, Kim H-W, Fu X, Leong KW. Advanced drug delivery systems and artificial skin grafts for skin wound healing. Adv Drug Deliv Rev. 2019;146:209-239.

15. Eming SA, Martin P, Tomic-Canic M. Wound repair and regeneration: mechanisms, signaling, and translation. Sci Transl Med. 2014;6(265):265sr6. doi:10.1126/scitranslmed.3009337

16. André-Lévigne D, Modarressi A, Pepper MS, Pittet-Cuénod B. Reactive oxygen species and NOX enzymes are emerging as key players in cutaneous wound repair. Int J Mol Sci. 2017;18 (10):2149. doi:10.3390/ijms18102149

17. Berthet M, Gauthier Y, Lacroix C, Verrier B, Monge C. Nanoparticle-based dressing: the future of wound treatment? Trends Biotechnol. 2017;35(8):770-784. doi:10.1016/j. tibtech.2017.05.005

18. Kalluri R, LeBleu VS. The biology, function, and biomedical applications of exosomes. Science. 2020;367(6478):eaau6977. doi:10.1126/science.aau6977

19. Rani S, Ritter T. The exosome - a naturally secreted nanoparticle and its application to wound healing. Adv Mater. 2016;28 (27):5542-5552. doi:10.1002/adma.201504009 
20. Casado-Díaz A, Quesada-Gómez JM, Dorado G. Extracellular vesicles derived from mesenchymal stem cells (MSC) in regenerative medicine: applications in skin wound healing. Front Bioeng Biotechnol. 2020;8:146. doi:10.3389/fbioe.2020.00146

21. Xie L, Wang J, Zhang Y, et al. The effects of local injection of exosomes derived from BMSCs on random skin flap in rats. Am $J$ Transl Res. 2019;11(11):7063-7073.

22. Martin P. Wound healing-aiming for perfect skin regeneration. Science. 1997;276(5309):75-81. doi:10.1126/science.276.5309.75

23. Stejskalová A, Almquist BD. Using biomaterials to rewire the process of wound repair. Biomater Sci. 2017;5(8):1421-1434. doi:10.1039/C7BM00295E

24. Macrae FL, Duval C, Papareddy P, et al. A fibrin biofilm covers blood clots and protects from microbial invasion. J Clin Invest. 2018;128(8):3356-3368. doi:10.1172/JCI98734

25. Opneja A, Kapoor S, Stavrou EX. Contribution of platelets, the coagulation and fibrinolytic systems to cutaneous wound healing. Thromb Res. 2019;179:56-63. doi:10.1016/j. thromres.2019.05.001

26. Sun J, Hua B, Livingston EW, et al. Abnormal joint and bone wound healing in hemophilia mice is improved by extending factor IX activity after hemarthrosis. Blood. 2017;129 (15):2161-2171. doi:10.1182/blood-2016-08-734053

27. Ishihara J, Ishihara A, Starke RD, et al. The heparin binding domain of von Willebrand factor binds to growth factors and promotes angiogenesis in wound healing. Blood. 2019;133 (24):2559-2569. doi:10.1182/blood.2019000510

28. Brazil JC, Quiros M, Nusrat A, Parkos CA. Innate immune cell-epithelial crosstalk during wound repair. J Clin Invest. 2019;129(8):2983-2993. doi:10.1172/JCI124618

29. Elliott MR, Koster KM, Murphy PS. Efferocytosis signaling in the regulation of macrophage inflammatory responses. J Immunol. 2017;198(4):1387-1394. doi:10.4049/jimmunol.1601520

30. Doni A, Garlanda C, Mantovani A. Innate immunity, hemostasis and matrix remodeling: PTX3 as a link. Semin Immunol. 2016;28 (6):570-577. doi:10.1016/j.smim.2016.10.012

31. Kanno E, Kawakami K, Ritsu M, et al. Wound healing in skin promoted by inoculation with Pseudomonas aeruginosa PAO1: the critical role of tumor necrosis factor- $\alpha$ secreted from infiltrating neutrophils. Wound Repair Regen. 2011;19(5):608-621. doi:10.1111/j.1524-475X.2011.00721.x

32. Landén NX, Li D, Ståhle M. Transition from inflammation to proliferation: a critical step during wound healing. Cell Mol Life Sci. 2016;73(20):3861-3885.

33. Li D, Wang A, Liu X, et al. MicroRNA-132 enhances transition from inflammation to proliferation during wound healing. J Clin Invest. 2015;125(8):3008-3026. doi:10.1172/JCI79052

34. Mahmoudi S, Mancini E, Xu L, et al. Heterogeneity in old fibroblasts is linked to variability in reprogramming and wound healing. Nature. 2019;574(7779):553-558. doi:10.1038/s41586019-1658-5

35. Driskell RR, Watt FM. Understanding fibroblast heterogeneity in the skin. Trends Cell Biol. 2015;25(2):92-99. doi:10.1016/j. tcb.2014.10.001

36. Gurtner GC, Werner S, Barrandon Y, Longaker MT. Wound repair and regeneration. Nature. 2008;453(7193):314-321. doi:10.1038/ nature07039

37. Keane TJ, Horejs CM, Stevens MM. Scarring vs. functional healing: matrix-based strategies to regulate tissue repair. $A d v$ Drug Deliv Rev. 2018;129:407-419. doi:10.1016/j. addr.2018.02.002

38. Mirastschijski U, Lupše B, Maedler K, et al. Matrix metalloproteinase-3 is key effector of TNF- $\alpha$-induced collagen degradation in skin. Int $J$ Mol Sci. 2019;20(20):5234. doi:10.3390/ ijms20205234
39. Hu MS, Hong WX, Januszyk M, et al. Pathway analysis of gene expression in murine fetal and adult wounds. Adv Wound Care. 2018;7(8):262-275. doi:10.1089/wound.2017.0779

40. Geim AK, Novoselov KS. The rise of graphene. Nat Mater. 2007;6(3):183-191. doi:10.1038/nmat1849

41. Allen MJ, Tung VC, Kaner RB. Honeycomb carbon: a review of graphene. Chem Rev. 2010;110(1):132-145. doi:10.1021/cr900070d

42. Yan J-A, Xian L, Chou MY. Structural and electronic properties of oxidized graphene. Phys Rev Lett. 2009;103(8):086802. doi:10.1103/PhysRevLett.103.086802

43. Tene T, Tubon Usca G, Guevara M, et al. Toward large-scale production of oxidized graphene. Nanomaterials. 2020;10(2):279. doi:10.3390/nano10020279

44. Luong DX, Bets KV, Algozeeb WA, et al. Gram-scale bottom-up flash graphene synthesis. Nature. 2020;577(7792):647-651. doi:10.1038/s41586-020-1938-0

45. Novoselov KS, Jiang D, Schedin F, et al. Two-dimensional atomic crystals. Proc Natl Acad Sci USA. 2005;102 (30):10451-10453. doi:10.1073/pnas.0502848102

46. Meyer JC, Geim AK, Katsnelson MI, Novoselov KS, Booth TJ, Roth S. The structure of suspended graphene sheets. Nature. 2007;446(7131):60-63. doi:10.1038/nature05545

47. Lotya M, Hernandez Y, King PJ, et al. Liquid phase production of graphene by exfoliation of graphite in surfactant/water solutions. J Am Chem Soc. 2009;131(10):3611-3620. doi:10.1021/ja807449u

48. Hernandez Y, Nicolosi V, Lotya M, et al. High-yield production of graphene by liquid-phase exfoliation of graphite. Nat Nanotechnol. 2008;3(9):563-568. doi:10.1038/nnano.2008.215

49. Lange RZ, Synnatschke K, Qi H, et al. Enriching and quantifying porous single layer 2D polymers by exfoliation of chemically modified van der Waals crystals. Angew Chem Int Ed Engl. 2020;59(14):5683-5695. doi:10.1002/anie.201912705

50. Zhu Y, Murali S, Cai W, et al. Graphene and graphene oxide: synthesis, properties, and applications. Adv Mater. 2010;22 (35):3906-3924. doi:10.1002/adma.201001068

51. Yu H, Zhang B, Bulin C, Li R, Xing R. High-efficient synthesis of graphene oxide based on improved hummers method. Sci Rep. 2016;6:36143. doi:10.1038/srep36143

52. Kim KS, Zhao Y, Jang H, et al. Large-scale pattern growth of graphene films for stretchable transparent electrodes. Nature. 2009;457(7230):706-710. doi:10.1038/nature07719

53. Khan A, Islam SM, Ahmed S, et al. Direct CVD growth of graphene on technologically important dielectric and semiconducting substrates. Adv Sci. 2018;5(11):1800050. doi:10.1002/ advs.201800050

54. Li Y, Li Z, Li Q, et al. Direct synthesis of graphene dendrites on $\mathrm{SiO} / \mathrm{Si}$ substrates by chemical vapor deposition. Nanoscale Res Lett. 2020;15(1):16. doi:10.1186/s11671-020-3245-y

55. Tracy J, Zietz O, Olson S, Jiao J. Plasma-enhanced chemical vapor deposition of acetylene on codeposited bimetal catalysts increasing graphene sheet continuity under low-temperature growth conditions. Nanoscale Res Lett. 2019;14(1):335. doi:10.1186/s11671-019-3156-y

56. Lee C, Wei X, Kysar JW, Hone J. Measurement of the elastic properties and intrinsic strength of monolayer graphene. Science. 2008;321(5887):385-388. doi:10.1126/science.1157996

57. Chen Y, Wang Y, Su T, et al. Self-healing polymer composites based on hydrogen bond reinforced with graphene oxide. ES Mater Manuf. 2019;4:31-37.

58. Chen Y, Guo Z, Das R, Jiang Q. Starch-based carbon nanotubes and graphene: preparation, properties and applications. ES Food Agroforestry. 2020;2:13-21.

59. Zhao H, Ding R, Zhao X, et al. Graphene-based nanomaterials for drug and/or gene delivery, bioimaging, and tissue engineering. Drug Discov Today. 2017;22(9):1302-1317. doi:10.1016/j. drudis.2017.04.002 
60. Li J, Zhou C, Luo C, et al. N-acetyl cysteine-loaded graphene oxide-collagen hybrid membrane for scarless wound healing. Theranostics. 2019;9(20):5839-5853. doi:10.7150/thno.34480

61. De Marco P, Zara S, De Colli M, et al. Graphene oxide improves the biocompatibility of collagen membranes in an in vitro model of human primary gingival fibroblasts. Biomed Mater. 2017;12 (5):055005. doi:10.1088/1748-605X/aa7907

62. Liu S, Zhou C, Mou S, et al. Biocompatible graphene oxide-collagen composite aerogel for enhanced stiffness and in situ bone regeneration. Mater Sci Eng C Mater Biol Appl. 2019;105:110137. doi:10.1016/j.msec.2019.110137

63. Donskyi IS, Azab W, Cuellar-Camacho JL, et al. Functionalized nanographene sheets with high antiviral activity through synergistic electrostatic and hydrophobic interactions. Nanoscale. 2019;11(34):15804-15809. doi:10.1039/C9NR05273A

64. Wychowaniec JK, Iliut M, Zhou M, et al. Designing peptide/ graphene hybrid hydrogels through fine-tuning of molecular interactions. Biomacromolecules. 2018;19(7):2731-2741. doi:10.1021/acs.biomac.8b00333

65. Yousefi M, Dadashpour M, Hejazi M, et al. Anti-bacterial activity of graphene oxide as a new weapon nanomaterial to combat multidrug-resistance bacteria. Mater Sci Eng C Mater Biol Appl. 2017;74:568-581. doi:10.1016/j.msec.2016.12.125

66. Chen J, Dai F, Zhang L, et al. Molecular insights into the dispersion stability of graphene oxide in mixed solvents: theoretical simulations and experimental verification. $J$ Colloid Interface Sci. 2020;571:109-117. doi:10.1016/j.jcis.2020.03.036

67. Tan KH, Sattari S, Donskyi IS, et al. Functionalized 2D nanomaterials with switchable binding to investigate graphene-bacteria interactions. Nanoscale. 2018;10(20):9525-9537. doi:10.1039/ C8NR01347K

68. Kim TI, Kwon B, Yoon J, et al. Antibacterial activities of graphene oxide-molybdenum disulfide nanocomposite films. ACS Appl Mater Interfaces. 2017;9(9):7908-7917. doi:10.1021/ acsami.6b12464

69. Qi Z, Bharate P, Lai C-H, et al. Multivalency at interfaces: supramolecular carbohydrate-functionalized graphene derivatives for bacterial capture, release, and disinfection. Nano Lett. 2015;15 (9):6051-6057. doi:10.1021/acs.nanolett.5b02256

70. Balandin AA, Ghosh S, Bao W, et al. Superior thermal conductivity of single-layer graphene. Nano Lett. 2008;8(3):902-907. doi:10.1021/n10731872

71. Guo W, Wang S, Yu X, et al. Construction of a 3D rGO-collagen hybrid scaffold for enhancement of the neural differentiation of mesenchymal stem cells. Nanoscale. 2016;8(4):1897-1904. doi:10.1039/C5NR06602F

72. Lasocka I, Szulc-Dąbrowska L, Skibniewski M, et al. Biocompatibility of pristine graphene monolayer: scaffold for fibroblasts. Toxicol in Vitro. 2018;48:276-285. doi:10.1016/j. tiv.2018.01.028

73. Cao Y, Li D, Zhao M, Gong H, Wan R, Gu H. N implantation induce cytocompatibility of shape-controlled three-dimensional self-assembly graphene. Nanomedicine. 2017;12(18):2245-2255. doi:10.2217/nnm-2017-0086

74. Shie MY, Chiang WH, Chen IWP, Liu WY, Chen YW. Synergistic acceleration in the osteogenic and angiogenic differentiation of human mesenchymal stem cells by calcium silicate-graphene composites. Mater Sci Eng C Mater Biol Appl. 2017;73:726-735. doi:10.1016/j.msec.2016.12.071

75. Olate-Moya F, Arens L, Wilhelm M, Mateos-Timoneda MA, Engel E, Palza H. Chondroinductive alginate-based hydrogels having graphene oxide for $3 \mathrm{D}$ printed scaffold fabrication. ACS Appl Mater Interfaces. 2020;12(4):4343-4357. doi:10.1021/ acsami.9b22062
76. Bhusari SA, Sharma V, Bose S, Basu B. HDPE/UHMWPE hybrid nanocomposites with surface functionalized graphene oxide towards improved strength and cytocompatibility. $J R$ Soc Interface. 2019;16(150):20180273. doi:10.1098/rsif.2018.0273

77. Nyambat B, Chen C-H, Wong P-C, Chiang C-W, Satapathy MK, Chuang EY. Genipin-crosslinked adipose stem cell derived extracellular matrix-nano graphene oxide composite sponge for skin tissue engineering. $J$ Mater Chem B. 2018;6(6):979-990. doi:10.1039/C7TB02480K

78. Wang K, Ruan J, Song H, et al. Biocompatibility of graphene oxide. Nanoscale Res Lett. 2011;6(1):8.

79. Pelin M, Fusco L, Martín C, et al. Graphene and graphene oxide induce ROS production in human HaCaT skin keratinocytes: the role of xanthine oxidase and NADH dehydrogenase. Nanoscale. 2018;10(25):11820-11830. doi:10.1039/C8NR02933D

80. Geng H, Wang T, Cao H, Zhu H, Di Z, Liu X. Antibacterial ability, cytocompatibility and hemocompatibility of fluorinated graphene. Colloids Surf B Biointerfaces. 2019;173:681-688. doi:10.1016/j.colsurfb.2018.10.050

81. Guo S, Nishina Y, Bianco A, Ménard-Moyon C. A flexible method for covalent double functionalization of graphene oxide. Angew Chem Int Ed Engl. 2020;59(4):1542-1547. doi:10.1002/anie.201913461

82. Guo Y, Chen Y, Han P, et al. Biocompatible chitosan-carbon nanocage hybrids for sustained drug release and highly efficient laser and microwave co-irradiation induced cancer therapy. Acta Biomaterialia. 2020;103:237-246. doi:10.1016/j.actbio.2019.12.010

83. Dos Reis SRR, Pinto SR, de Menezes FD, et al. Senescence and the impact on biodistribution of different nanosystems: the discrepancy on tissue deposition of graphene quantum dots, polycaprolactone nanoparticle and magnetic mesoporous silica nanoparticles in young and elder animals. Pharm Res. 2020;37 (3):40. doi:10.1007/s11095-019-2754-9

84. Fusco L, Garrido M, Martín C, et al. Skin irritation potential of graphene-based materials using a non-animal test. Nanoscale. 2020;12(2):610-622. doi:10.1039/C9NR06815E

85. Duch MC, Budinger GRS, Liang YT, et al. Minimizing oxidation and stable nanoscale dispersion improves the biocompatibility of graphene in the lung. Nano Lett. 2011;11(12):5201-5207. doi:10.1021/n1202515a

86. Amrollahi-Sharifabadi M, Koohi MK, Zayerzadeh E, Hablolvarid MH, Hassan J, Seifalian AM. In vivo toxicological evaluation of graphene oxide nanoplatelets for clinical application. Int J Nanomedicine. 2018;13:4757-4769. doi:10.2147/IJN.S168731

87. Kenry LW, Loh KP, Lim CT. When stem cells meet graphene: opportunities and challenges in regenerative medicine. Biomaterials. 2018;155:236-250. doi:10.1016/j.biomaterials.2017.10.004

88. Li Y, Feng L, Shi X, et al. Surface coating-dependent cytotoxicity and degradation of graphene derivatives: towards the design of non-toxic, degradable nano-graphene. Small. 2014;10 (8):1544-1554. doi:10.1002/smll.201303234

89. Fallatah H, Elhaneid M, Ali-Boucetta H, Overton TW, El Kadri H, Gkatzionis K. Antibacterial effect of graphene oxide (GO) nano-particles against Pseudomonas putida biofilm of variable age. Environ Sci Pollut Res Int. 2019;26(24):25057-25070. doi:10.1007/s11356-019-05688-9

90. Pulingam T, Thong KL, Appaturi JN, et al. Synergistic antibacterial actions of graphene oxide and antibiotics towards bacteria and the toxicological effects of graphene oxide on human epidermal keratinocytes. Eur J Pharm Sci. 2020;142:105087. doi:10.1016/j.ejps.2019.105087

91. Hussein KH, Abdelhamid HN, Zou X, Woo HM. Ultrasonicated graphene oxide enhances bone and skin wound regeneration. Mater Sci Eng C Mater Biol Appl. 2019;94:484-492. doi:10.1016/j.msec.2018.09.051 
92. Cheng Y, Chang Y, Feng Y, et al. Hierarchical acceleration of wound healing through intelligent nanosystem to promote multiple stages. ACS Appl Mater Interfaces. 2019;11 (37):33725-33733. doi:10.1021/acsami.9b13267

93. Thangavel P, Kannan R, Ramachandran B, Moorthy G, Suguna L, Muthuvijayan V. Development of reduced graphene oxide (rGO)-isabgol nanocomposite dressings for enhanced vascularization and accelerated wound healing in normal and diabetic rats. $J$ Colloid Interface Sci. 2018;517:251-264. doi:10.1016/j. jcis.2018.01.110

94. Esmaeili E, Eslami-Arshaghi T, Hosseinzadeh S, et al. The biomedical potential of cellulose acetate/polyurethane nanofibrous mats containing reduced graphene oxide/silver nanocomposites and curcumin: antimicrobial performance and cutaneous wound healing. Int J Biol Macromol. 2020;152:418-427. doi:10.1016/j. ijbiomac.2020.02.295

95. Rehman SRU, Augustine R, Zahid AA, Ahmed R, Tariq M, Hasan A. Reduced graphene oxide incorporated GelMA hydrogel promotes angiogenesis for wound healing applications. Int $J$ Nanomedicine. 2019;14:9603-9617. doi:10.2147/IJN. S218120

96. Liang Y, Zhao X, Hu T, et al. Adhesive hemostatic conducting injectable composite hydrogels with sustained drug release and photothermal antibacterial activity to promote full-thickness skin regeneration during wound healing. Small. 2019;15(12): e1900046. doi:10.1002/smll.201900046

97. Tang P, Han L, Li P, et al. Mussel-inspired electroactive and antioxidative scaffolds with incorporation of polydopamine-reduced graphene oxide for enhancing skin wound healing. ACS Appl Mater Interfaces. 2019;11 (8):7703-7714. doi:10.1021/acsami.8b18931

98. Yan X, Fang -W-W, Xue J, et al. Thermoresponsive forming hydrogel with sol-gel irreversibility for effective methicillin-resistant infected wound healing. ACS Nano. 2019;13(9):10074-10084. doi:10.1021/acsnano.9b02845

99. Mellado C, Figueroa T, Baez R, et al. Development of graphene oxide composite aerogel with proanthocyanidins with hemostatic properties as a delivery system. ACS Appl Mater Interfaces. 2018;10(9):7717-7729. doi:10.1021/acsami.7b16084

100. Xue J, Wang X, Wang E, Li T, Chang J, Wu C. Bioinspired multifunctional biomaterials with hierarchical microstructure for wound dressing. Acta Biomaterialia. 2019;100:270-279. doi:10.1016/j.actbio.2019.10.012

101. Barahuie F, Saifullah B, Dorniani D, et al. Graphene oxide as a nanocarrier for controlled release and targeted delivery of an anticancer active agent, chlorogenic acid. Mater Sci Eng C Mater Biol Appl. 2017;74:177-185. doi:10.1016/j.msec.2016.11.114

102. Lim E-B, Vy TA, Lee S-W. Comparative release kinetics of small drugs (ibuprofen and acetaminophen) from multifunctional mesoporous silica nanoparticles. $J$ Mater Chem B. 2020;8 (10):2096-2106. doi:10.1039/C9TB02494H

103. Shirvalilou S, Khoei S, Khoee S, Raoufi NJ, Karimi MR, ShakeriZadeh A. Development of a magnetic nano-graphene oxide carrier for improved glioma-targeted drug delivery and imaging: in vitro and in vivo evaluations. Chem Biol Interact. 2018;295:295. doi:10.1016/j.cbi.2018.08.027

104. Wang L, Yu D, Dai R, et al. PEGylated doxorubicin cloaked nano-graphene oxide for dual-responsive photochemical therapy. Int J Pharm. 2019;557:66-73. doi:10.1016/j.ijpharm.2018.12.037

105. Liu T, Dan W, Dan N, Liu X, Liu X, Peng X. A novel graphene oxide-modified collagen-chitosan bio-film for controlled growth factor release in wound healing applications. Mater Sci Eng C Mater Biol Appl. 2017;77:202-211. doi:10.1016/j. msec.2017.03.256
106. Vinothini K, Rajendran NK, Munusamy MA, Alarfaj AA, Rajan M. Development of biotin molecule targeted cancer cell drug delivery of doxorubicin loaded $\kappa$-carrageenan grafted graphene oxide nanocarrier. Mater Sci Eng C Mater Biol Appl. 2019;100:676-687. doi:10.1016/j.msec.2019.03.011

107. Rasoulzadeh M, Namazi H. Carboxymethyl cellulose/graphene oxide bio-nanocomposite hydrogel beads as anticancer drug carrier agent. Carbohydr Polym. 2017;168:320-326. doi:10.1016/j. carbpol.2017.03.014

108. Borandeh S, Abdolmaleki A, Abolmaali SS, Tamaddon AM. Synthesis, structural and in-vitro characterization of $\beta$ cyclodextrin grafted L-phenylalanine functionalized graphene oxide nanocomposite: a versatile nanocarrier for $\mathrm{pH}$-sensitive doxorubicin delivery. Carbohydr Polym. 2018;201:151-161. doi:10.1016/j.carbpol.2018.08.064

109. Wu D, Samanta A, Srivastava RK, Hakkarainen M. Starch-derived nanographene oxide paves the way for electrospinnable and bioactive starch scaffolds for bone tissue engineering. Biomacromolecules. 2017;18(5):1582-1591. doi:10.1021/acs.biomac.7b00195

110. Ligorio C, Zhou M, Wychowaniec JK, et al. Graphene oxide containing self-assembling peptide hybrid hydrogels as a potential 3D injectable cell delivery platform for intervertebral disc repair applications. Acta Biomaterialia. 2019;92:92-103. doi:10.1016/j.actbio.2019.05.004

111. Kazantseva J, Ivanov R, Gasik M, Neuman T, Hussainova I. Graphene-augmented nanofiber scaffolds trigger gene expression switching of four cancer cell types. ACS Biomater Sci Eng. 2018;4(5):1622-1629. doi:10.1021/acsbiomaterials.8b00228

112. Wang J, Cui C, Nan H, et al. Graphene sheet-induced global maturation of cardiomyocytes derived from human induced pluripotent stem cells. ACS Appl Mater Interfaces. 2017;9 (31):25929-25940. doi:10.1021/acsami.7b08777

113. Tonellato M, Piccione M, Gasparotto M, et al. Commitment of autologous human multipotent stem cells on biomimetic poly-L-lactic acid-based scaffolds is strongly influenced by structure and concentration of carbon nanomaterial. Nanomaterials. 2020;10(3):415. doi:10.3390/nano10030415

114. Saravanabhavan SS, Rethinasabapathy M, Zsolt S, et al. Graphene oxide functionalized with chitosan based nanoparticles as a carrier of siRNA in regulating Bcl-2 expression on Saos-2 \& MG-63 cancer cells and its inflammatory response on bone marrow derived cells from mice. Mater Sci Eng C Mater Biol Appl. 2019;99:1459-1468. doi:10.1016/j.msec.2019.02.047

115. Imani R, Prakash S, Vali H, Faghihi S. Polyethylene glycol and octa-arginine dual-functionalized nanographene oxide: an optimization for efficient nucleic acid delivery. Biomater Sci. 2018;6 (6):1636-1650. doi:10.1039/C8BM00058A

116. Hamdan S, Pastar I, Drakulich S, et al. Nanotechnology-driven therapeutic interventions in wound healing: potential uses and applications. ACS Cent Sci. 2017;3(3):163-175. doi:10.1021/acscentsci.6b00371

117. Yang L, Zhai Y, Hao Y, Zhu Z, Cheng G. The regulatory functionality of exosomes derived from hUMSCs in 3D culture for alzheimer's disease therapy. Small. 2020;16(3):e1906273. doi:10.1002/smll.201906273

118. Arnold AM, Holt BD, Daneshmandi L, Laurencin CT, Sydlik SA. Phosphate graphene as an intrinsically osteoinductive scaffold for stem cell-driven bone regeneration. Proc Natl Acad Sci USA. 2019;116(11):4855-4860. doi:10.1073/ pnas. 1815434116

119. Zhang B, Wei P, Zhou Z, Wei T. Interactions of graphene with mammalian cells: molecular mechanisms and biomedical insights. Adv Drug Deliv Rev. 2016;105(Pt B):145-162. doi:10.1016/j. addr.2016.08.009 
120. Chu J, Shi P, Yan W, et al. PEGylated graphene oxide-mediated quercetin-modified collagen hybrid scaffold for enhancement of MSCs differentiation potential and diabetic wound healing. Nanoscale. 2018;10(20):9547-9560. doi:10.1039/C8NR02538J

121. Shen H, Lin H, Sun AX, et al. Acceleration of chondrogenic differentiation of human mesenchymal stem cells by sustained growth factor release in 3D graphene oxide incorporated hydrogels. Acta Biomaterialia. 2020;105:44-55. doi:10.1016/j. actbio.2020.01.048

122. Phinney DG, Pittenger MF. Concise review: MSC-derived exosomes for cell-free therapy. Stem Cells. 2017;35(4):851-858. doi:10.1002/stem.2575

123. Li X, Liu L, Yang J, et al. Exosome derived from human umbilical cord mesenchymal stem cell mediates MiR-181c attenuating burn-induced excessive inflammation. EBioMedicine. 2016;8:72-82. doi:10.1016/j.ebiom.2016.04.030

124. Hu L, Wang J, Zhou X, et al. Exosomes derived from human adipose mesenchymal stem cells accelerates cutaneous wound healing via optimizing the characteristics of fibroblasts. Sci Rep. 2016;6:32993. doi:10.1038/srep32993

125. Zhang W, Bai X, Zhao B, et al. Cell-free therapy based on adipose tissue stem cell-derived exosomes promotes wound healing via the PI3K/Akt signaling pathway. Exp Cell Res. 2018;370 (2):333-342. doi:10.1016/j.yexcr.2018.06.035

126. Zhang B, Shi Y, Gong A, et al. HucMSC exosome-delivered 14-

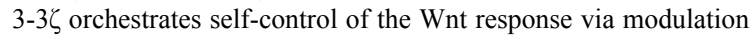
of YAP during cutaneous regeneration. Stem Cells. 2016;34 (10):2485-2500. doi:10.1002/stem.2432

127. Dalirfardouei R, Jamialahmadi K, Jafarian AH, Mahdipour E. Promising effects of exosomes isolated from menstrual blood-derived mesenchymal stem cell on wound-healing process in diabetic mouse model. J Tissue Eng Regen Med. 2019;13 (4):555-568. doi:10.1002/term.2799

128. Chen CY, Rao SS, Ren L, et al. Exosomal DMBT1 from human urine-derived stem cells facilitates diabetic wound repair by promoting angiogenesis. Theranostics. 2018;8(6):1607-1623. doi:10.7150/ thno. 22958

129. Ding J, Wang X, Chen B, Zhang J, Xu J. Exosomes derived from human bone marrow mesenchymal stem cells stimulated by deferoxamine accelerate cutaneous wound healing by promoting angiogenesis. Biomed Res Int. 2019;2019:9742765. doi:10.1155/2019/9742765

130. Yang K, Li D, Wang M, et al. Exposure to blue light stimulates the proangiogenic capability of exosomes derived from human umbilical cord mesenchymal stem cells. Stem Cell Res Ther. 2019;10(1):358. doi:10.1186/s13287-019-1472-x

131. Li B, Luan S, Chen J, et al. The MSC-derived exosomal lncRNA H19 promotes wound healing in diabetic foot ulcers by upregulating PTEN via microRNA-152-3p. Mol Ther Nucleic Acids. 2020;19:814-826. doi:10.1016/j.omtn.2019.11.034

132. Zhang K, Zhao X, Chen X, et al. Enhanced therapeutic effects of mesenchymal stem cell-derived exosomes with an injectable hydrogel for hindlimb ischemia treatment. ACS Appl Mater Interfaces. 2018;10(36):30081-30091. doi:10.1021/acsami.8b08449

133. Cheng N-C, Lin W-J, Ling T-Y, Young T-H. Sustained release of adipose-derived stem cells by thermosensitive chitosan/gelatin hydrogel for therapeutic angiogenesis. Acta Biomaterialia. 2017;51:258-267. doi:10.1016/j.actbio.2017.01.060

134. Tao S-C, Guo S-C, Li M, Ke Q-F, Guo Y-P, Zhang C-Q. Chitosan wound dressings incorporating exosomes derived from microRNA-126-overexpressing synovium mesenchymal stem cells provide sustained release of exosomes and heal full-thickness skin defects in a diabetic rat model. Stem Cells Transl Med. 2017;6(3):736-747. doi:10.5966/ sctm.2016-0275
135. Shi Q, Qian Z, Liu D, et al. GMSC-derived exosomes combined with a chitosan/silk hydrogel sponge accelerates wound healing in a diabetic rat skin defect model. Front Physiol. 2017;8:904. doi:10.3389/fphys.2017.00904

136. Shafei S, Khanmohammadi M, Heidari R, et al. Exosome loaded alginate hydrogel promotes tissue regeneration in full-thickness skin wounds: an in-vivo study. J Biomed Mater Res A. 2020;108 (3):545-556. doi:10.1002/jbm.a.36835

137. Mol EA, Lei Z, Roefs MT, et al. Injectable supramolecular ureidopyrimidinone hydrogels provide sustained release of extracellular vesicle therapeutics. Adv Healthc Mater. 2019;8(20): e1900847. doi:10.1002/adhm.201900847

138. Wang C, Wang M, Xu T, et al. Engineering bioactive self-healing antibacterial exosomes hydrogel for promoting chronic diabetic wound healing and complete skin regeneration. Theranostics. 2019;9(1):65-76. doi:10.7150/thno.29766

139. Wang M, Wang C, Chen M, et al. Efficient angiogenesis-based diabetic wound healing/skin reconstruction through bioactive antibacterial adhesive ultraviolet shielding nanodressing with exosome release. ACS Nano. 2019;13:10279-10293.

140. Liu X, Yang Y, Li Y, et al. Integration of stem cell-derived exosomes with in situ hydrogel glue as a promising tissue patch for articular cartilage regeneration. Nanoscale. 2017;9 (13):4430-4438. doi:10.1039/C7NR00352H

141. Rodrigues M, Kosaric N, Bonham CA, Gurtner GC. Wound healing: a cellular perspective. Physiol Rev. 2019;99 (1):665-706. doi:10.1152/physrev.00067.2017

142. Wang C, Liang C, Wang R, et al. The fabrication of a highly efficient self-healing hydrogel from natural biopolymers loaded with exosomes for the synergistic promotion of severe wound healing. Biomater Sci. 2019;8(1):313-324. doi:10.1039/ C9BM01207A

143. Chen P, Zheng L, Wang Y, et al. Desktop-stereolithography 3D printing of a radially oriented extracellular matrix/mesenchymal stem cell exosome bioink for osteochondral defect regeneration. Theranostics. 2019;9(9):2439-2459. doi:10.7150/thno.31 017

144. Liu X, Miller AL, Park S, et al. Two-dimensional black phosphorus and graphene oxide nanosheets synergistically enhance cell proliferation and osteogenesis on 3D printed scaffolds. ACS Appl Mater Interfaces. 2019;11(26):23558-23572. doi:10.1021/ acsami.9b04121

145. Choe G, Oh S, Seok JM, Park SA, Lee JY. Graphene oxide/ alginate composites as novel bioinks for three-dimensional mesenchymal stem cell printing and bone regeneration applications. Nanoscale. 2019;11(48):23275-23285. doi:10.1039/C9NR07643C

146. Liu S, Bastola AK, Li L. A 3D printable and mechanically robust hydrogel based on alginate and graphene oxide. ACS Appl Mater Interfaces. 2017;9(47):41473-41481. doi:10.1021/ acsami.7b13534

147. Tiwari S, Patil R, Dubey SK, Bahadur P. Graphene nanosheets as reinforcement and cell-instructive material in soft tissue scaffolds. Adv Colloid Interface Sci. 2020;281:102167. doi:10.1016/j. cis. 2020.102167

148. Patel DB, Luthers CR, Lerman MJ, Fisher JP, Jay SM. Enhanced extracellular vesicle production and ethanol-mediated vascularization bioactivity via a 3D-printed scaffold-perfusion bioreactor system. Acta Biomaterialia. 2019;95:236-244. doi:10.1016/j. actbio.2018.11.024

149. Phan J, Kumar P, Hao D, Gao K, Farmer D, Wang A. Engineering mesenchymal stem cells to improve their exosome efficacy and yield for cell-free therapy. $J$ Extracell Vesicles. 2018;7 (1):1522236. doi:10.1080/20013078.2018.1522236 
150. Feng Y, Chen Q, Yin Q, Pan G, Tu Z, Liu L. Reduced graphene oxide functionalized with gold nanostar nanocomposites for synergistically killing bacteria through intrinsic antimicrobial activity and photothermal ablation. ACS Appl Bio Mater. 2019;2 (2):747-756. doi:10.1021/acsabm.8b00608

151. An Y, Lin S, Tan X, et al. Exosomes from adipose-derived stem cells and application to skin wound healing. Cell Prolif. 2021; e12993. doi:10.1111/cpr.12993
152. Yang $\mathrm{S}$, Zhu B, Yin $\mathrm{P}$, et al. Integration of human umbilical cord mesenchymal stem cells-derived exosomes with hydroxyapatite-embedded hyaluronic acid-alginate hydrogel for bone regeneration. ACS Biomater Sci Eng. 2020;6(3):1590-1602. doi:10.1021/acsbiomaterials.9b01363

\section{Publish your work in this journal}

The International Journal of Nanomedicine is an international, peerreviewed journal focusing on the application of nanotechnology in diagnostics, therapeutics, and drug delivery systems throughout the biomedical field. This journal is indexed on PubMed Central, MedLine, CAS, SciSearch ${ }^{\mathbb{B}}$, Current Contents ${ }^{\mathbb{B}} /$ Clinical Medicine, $^{2}$
Journal Citation Reports/Science Edition, EMBase, Scopus and the Elsevier Bibliographic databases. The manuscript management system is completely online and includes a very quick and fair peer-review system, which is all easy to use. Visit http://www.dovepress.com/ testimonials.php to read real quotes from published authors.

Submit your manuscript here: https://www.dovepress.com/international-journal-of-nanomedicine-journal 